Historic, Archive Document

Do not assume content reflects current scientific knowledge, policies, or practices. 



\title{
Remarks
}

THE MORRIS NURSERIES were established in 1849. The Morris Nursery Co., was incorporated during the year 1906. Sales Office, 1133 Broadway, New York City, N. Y.

TO CORRESPONDENTS-We desire to thank the public for their liberal patronage, and solicit a continuance of the same. We aim to grow and supply the best of stock at the lowest prices consistent with the quality of stock offered. Where a large number of trees and plants are required we solicit a list of wants for special quotations.

LOCATION-The nurseries are located at West Chester, county seat of Chester County, in one of the richest and most fertile spots in Pennsylvania, having the richest soil and best climate to be found in this country for growing nursery stock; 117 miles west of New York City and 27 miles west of Philadelphia.

PRICES are f. o. b. nurseries, point of shipment. Stock furnished at special net prices in carload lots, freight charges to follow from point of shipment unless otherwise agreed upon.

SHIPPING-Spring season commences between March 15th and April 1st, and continues to June. Autumn season commences in September and ends with the freezing of the ground in December. Orders should be placed early to insure good stock and varieties wanted.

MISTAKES-We give most careful attention to the shipping of varieties as ordered and warrant our stock true to name, but we do not hold ourselves responsible to any greater extent than the original cost of the goods or replacing the same. Shipments are made when stock is in best condition for removal. Most deciduous trees and shrubs succeed well both in Spring and Autumn planting. Evergreens do better if planted in April or May.

REFERENCES-All orders from unknown parties must be accompanied by check, draft, express or money order, unless satisfactory reference is given. To avoid mistakes write the order plainly, giving both post office and shipping directions. All articles are at the risk of the purchaser after being delivered to the railroad or express companies.

COMPLAINTS will not receive consideration unless made upon receipt of the goods. By careful consideration of the wants of the public and strict attention to business we hope to receive a liberal share of your patronage.

GARDENERS-We have on file in our office names of competent gardeners wanting employment such as we can recommend to our patrons as trustworthy and reliable. There is no charge for registering or for the service.

LANDSCAPE ARCHITECT-We are prepared to furnish a first-class landscape architect and engineer to make surveys, draw plans and give estimates for the building of roads and walks and the planting of trees and plants required for such work. We solicit, correspondence from all who need such service.

\section{THE MORRIS NURSERY COMPANY}

\author{
1133 Broadway, New York City, N. Y.
}

Telephone: Watkins 0727 


\section{Deciduous, Shade and Or- namental Trees}

\section{ACER. Maple}

A. dasycarpum. Silver-leaved Maple. Light green foliage, silvery beneath; hardy, rapid grower.

8-10 ft., $\$ 12.50$ per 10 .

10-12 ft., $\$ 20.00$ per 10 .

$12-14$ ft., $\$ 30.00$ per 10 .

A. dasycarpum Wieri laciniatum. Wier's Cut-leaved Silver Maple. A beautiful variety with dissected foliage and long, drooping branches.

8-10 ft., $\$ 20.00$ per 10 .

10-12 ft., $\$ 30.00$ per 10 .

$12-14$ ft., $\$ 45.00$ per 10 .

A. Negundo. Ash-Leaved Maple, or Box Elder. A quick grower, hardy tree, with Ash-like foliage, well suited for seaside planting.

8-10 ft., $\$ 12.00$ per 10.

10-12 ft., $\$ 20.00$ per 10.

A. platanoides. Norway Maple. Handsome European tree; large, symmetrical, with shinning deep green leaves; fine shade tree.

10-12 ft., $\$ 40.00$ per 10 .

12-14 ft., $\$ 50.00$ per 10 .

$14-16$ ft., $\$ 60.00$ per 10 .

A. pseudo-platanus. Sycamore Maple. A familiar street tree; large foliage and smooth bark.

$10 \mathrm{ft}$., $\$ 30.00$ per 10 .

10-12 ft., $\$ 35.00$ per 10 .

$12-14 \mathrm{ft} ., 2-2 \frac{1}{4}$ in. cal., $\$ 50.00$ per 10.

A. platanoides Schwedleri. Schwedler's Norway Maple. Young foliage purplish-erimson, changing to deep green in Autumn.

8-10 ft., $\$ 40.00$ per 10 .

12 ft., $\$ 50.00$ per 10 .

A. rubrum. Red, or Scarlet Maple. Deep red blossoms before the leaves in Spring; in Autumn the foliage turns to brilliant scarlet, very showy.

8-10 ft., $\$ 30.00$ per 10 .

10-12 ft., $\$ 40.00$ per 10 .

$12-14$ ft., $\$ 50.00$ per 10 .

14 ft., $\mathrm{X} \$ 60.00$ per 10 .

A. saccharinum. Sugar, or Rock Maple. A tree of stately pyramidal growth and large size; one of the finest trees for street or avenue.

8-10 ft., $\$ 25.00$ per 10 .

10-12 ft., $\$ 35.00$ per 10 .

$12-14$ ft., $\$ 50.00$ per 10 .

14 ft., X $\$ 60.00$ per 10.

A. tataricum, var. Ginnala. A beautiful hardy tree of moderate dwarf growth and foliage turns a bright red in Autumn; can be used as a substitute for Japan Maples.

4-5 ft., $\$ 17.00$ per 10.

5-6 ft., $\$ 26.00$ per 10 . 


\section{JAPAN MAPLES}

A. polymorphum atropurpureum. Dark Purple Leaved Maple. Fine cut-leaved; leaves dark reddish purple.

3 ft., $\$ 20.00$ each.

4 ft., $\$ 25.00$ each.

\section{AESCULUS. Horse Chestnut}

AE. Hippocastanum. European Horse Chestnut. Large, handsome tree, with beautiful, erect spikes of white flowers marked with red.

8 ft., $\$ 30.00$ per 10.

10-12 ft., $\$ 60.00$ per 10 .

12-14 ft., $\$ 70.00$ per 10 .

\section{AMELANCHIER. Mespilus}

A. Canadensis. Juneberry, or Service Tree. One of the best early flowering trees; covered with white flowers in April followed by small purplish fruit. $2-3$ ft., $\$ 7.50$ per 10 .

3-4 ft., $\$ 10.00$ per 10 .

\section{ARALIA. Angelica Tree}

A..spinosa. Hercules' Club. A native variety, very thorny and of striking appearance.

3-4 ft., $\$ 9.00$ per 10 .

4-5 ft., \$10.00 per 10 .

6-8 ft., $\$ 15.00$ per 10 .

\section{BETULA. Birch}

B. alba. White Birch. A graceful tree with silvery bark, habit erect at first, but droops with increasing growth.

4- 5 ft., $\$ 12.50$ per 10.

6- 8 ft., $\$ 17.00$ per 10 .

8-10 ft., $\$ 35.00$ per 10.

B. alba laciniata pendula. Cut-Leaved Weeping Bireh. A slender, weeping tree of vigorous growth, with silvery bark and finely cut foliage.

3-4 ft., $\$ 25.00$ per 10 .

4-6 ft., $\$ 30.00$ per 10.

6-8 ft., $\$ 45.00$ per 10 .

B. papyracea. Paper, or Canoe Birch. A tall and handsome tree with pure white bark.

4-6 ft., $\$ 17.00$ per 10 .

6-8 ft., $\$ 25.00$ per 10 .

\section{CARPINUS. Hornbeam}

C. americana. American Hornbeam. Similar in growth to Beech; excellent for hedges.

4-5 ft., $\$ 12.00$ per 10 .

6-8 ft., $\$ 20.00$ per 10 .

\section{CATALPA}

C. Bungei. Standard. A remarkable small tree forming a symmetrical round head as regular in shape as if sheared, valuable for formal planting.

2-Yr. heads, $\$ 40.00$ per 10.

C. speciosa. Hardy Western Catalpa. A very valuable species combining rapidity of growth, handsome foliage and flowers; perfectly hardy.

$10 \mathrm{ft}$., $\$ 12.50$ per 10 .

12 ft., $\$ 15.00$ per 10 .

12-14 ft., $\$ 25.00$ per 10 . 


\section{CELTIS. Nettle Tree}

C. occidentalis. American Nettle Tree, or Hackberry. Usually a small tree in the East but attains great size in the Southwest; general appearance of an Elm, but bears purplish berries.

$3-4$ ft., $\$ 9.00$ per 10 .

$4-5$ ft., $\$ 12.00$ per 10 .

6-8 ft., $\$ 20.00$ per 10 .

\section{CERCIDIPHYLLUM. Katsura Tree}

C. japonicum. An erect growing tree with rounded leaves, bright green, glaucous beneath.

3- 4 ft., $\$ 20.00$ per 10 .

8-10 ft., $\$ 50.00$ per 10 .

\section{CERCIS. Judas Tree}

C. canadensis. Red Bud. American Judas Tree. A small, handsome tree, with the naked branches covered in early Spring with reddish-pink flowers; heart-shaped leaves.

3-4 ft., $\$ 9.00$ per 10 .

4-5 ft. $\$ 12.50$ per 10 .

C. japonica. Oriental Judas Tree. Glossy leaves with whitish lines around margin, flowers larger than native variety.

2-3 ft., $\$ 12.50$ per 10 .

\section{CLADRASTIS. Yellow Wood Tree}

C. tinctoria. Yellow Wood. Foliage divided into 7 to 11 leaflets, branchlets terminating in large, loose panicles of creamy, fragrant flowers, peashaped.

$4-5$ ft., $\$ 25.00$ per 10 .

5-6 ft., $\$ 30.00$ per 10 .

\section{CORNUS. Dogwood}

C. florida. White Flowering Dogwood. Native Dogwood of graceful shape, white flowers before the leaves in Spring, followed by scarlet fruit.
3- 4 ft., $\$ 9.00$ per 10 .
4- 5 ft., $\$ 15.00$ per 10.
5. 6 ft., $\$ 20.00$ per 10 .
6- 7 ft., $\$ 22.00$ per 10.
8-10 ft., $\$ 35.00$ per 10 .
$10 \mathrm{ft} ., \$ 40.00$ per 10 .
12 ft., $\$ 50.00$ per 10.

C. florida rubra. Red Flowering Dogwood. Flowers

a bright rose, very ornamental.
2-3 ft., $\$ 20.00$ per 10 .
$3-4$ ft., $\$ 25.00$ per 10 .
5-6 ft., $\$ 40.00$ per 10 .
6-7 ft., $\$ 65.00$ per 10 .

\section{CRATAEGUS. Thorn}

c..coccinea. Scarlet-Fruited Thorn. Large, white flowers followed by scarlet fruit.

3-4 ft., $\$ 12.50$ per 10 .

4-5 ft., $\$ 15.00$ per 10 .

5-6 ft., $\$ 20.00$ per 10 .

c. flora alba plena. Double white.

4-5 ft., $\$ 25.00$ per 10 .

$6-8$ ft., $\$ 35.00$ per 10 . 
CRATAEGUS-Continued

C. Oxycantha. Common Hawthorn. The English hedge plant. Fragrant white flowers.

3-4 ft., $\$ 15.00$ per 10 .

4-5 ft. $\$ 20.00$ per 10 .

\section{FAGUS. Beech}

F. ferruginea. American Beech. Forest tree with smooth, light gray bark and toothed leaves which hang on through the Winter after drying; stately form.
3-4 ft., $\$ 20.00$ per 10 .
$4-5$ ft., $\$ 30.00$ per 10 .
7-8 ft., B \& B, $\$ 50.00$ per 10.

F. heterophylla. Fern-leaved Beech. Leaves delicately cut into fern-like form; round-headed tree, one of the finest Beeches.

$3-4$ ft., $\$ 30.00$ per 10 .

4-5 ft., $\$ 5.00$ each.

6-8 ft., B \& B, $\$ 10.00$ each.

F. purpurea Riversii. Rivers' Purple Beech. Foliage crimson in early Spring, dark purple in Summer.

The finest of purple-leaved trees.
3-4 ft., $\$ 5.00$ each.
4-5 ft., $\$ 6.00$ each.
6 ft., $\$ 10.00$ each.
8 ft., $\$ 15.00$ each.

F. sylvatica. European Beech. Broader and shorter leaves than the American; similar growth.
3- 4 ft., $\$ 2.50$ each.
4- 5 ft., $\$ 3.50$ each.
5- 6 ft., $\$ 4.50$ each.
6- 8 ft., $\$ 5.00$ each.
8-10 ft., $\$ 6.00$ each.

F. sylvatica pendula. Weeping Beech. Large, dark green leaves, twisting and tortuous spreading branches; a unique weeping tree.

4 ft., $\$ 5.00$ each.

\section{FRAXINUS. Ash}

F. americana. American White Ash. Large trees with light green leaves and pale yellowish green flowers.

8 ft., $\$ 12.50$ per 10 .

10 ft., $\$ 18.00$ per 10 .

12 ft., $\$ 30.00$ per 10 .

\section{GYMNOCLADUS. Coffee Tree}

G. canadensis. Kentucky Coffee Tree. A tall tree with compound leaflets, dark green with bluish tinge; rough bark; makes a handsome specimen.

3-4 ft., $\$ 1.50$ each.

4-5 ft., $\$ 2.00$ each.

5-6 ft., $\$ 3.00$ each.

\section{KOELREUTERIA. Varnish Tree}

K. paniculata. A very desirable Chinese tree; small, round-headed; showy panicles of golden yellow flowers in July, foliage turns yellow in Autumn.

$3-4$ ft., $\$ 12.50$ per 10 .

4-5 ft., $\$ 15.00$ per 10 .

6-8 ft., $\$ 20.00$ per 10 . 


\section{LARIX. Larch}

L. europaea. European Larch. A fast growing conifer pyramidal form, light green leaves, turning yellow in Fall.

$$
\begin{array}{lll}
3-4 & \text { ft., } \$ 15.50 \text { per } 10 . \\
4-5 & \text { ft., } \$ 20.00 \text { per } 10 .
\end{array}
$$

\section{LIQUIDAMBER. Sweet Gum}

L. Styraciflua. Sweet Gum. Beautiful tree with corky bark and star-shaped foliage, glossy green in Summer, changing to crimson, searlet, gold and bronze in Autumn.

$4-6$ ft., $\$ 20.00$ per 10.
$6-8$ ft., $\$ 30.00$ per 10.
10 ft., $\$ 6.00$ each.

\section{LIRIODENDRON. Tulip Tree}

L. Tulipfera. Tulip Tree, or Whitewood. A' superb native tree of spreading form and fine foliage, large yellow flowers marked with orange; difficult to transplant.

6- 8 ft., $\$ 20.00$ per 10 .

8-10 ft., $\$ 25.00$ per 10 .

10-12 ft., $\$ 40.00$ per 10 .

\section{MAGNOLIA}

M. glauca. Laurel Magnolia, Sweet Bay. Native variety, with handsome leaves and pure white, very fragrant flowers in Summer.

$2-3 \mathrm{ft}$., $\$ 3.00$ each.

3-4 ft., $\$ 4.00$ each.

M. tripetala. Umbrella Tree. Hardy, low growing tree, immense leaves, white flowers in June; fruit turns red in Autumn.

4-6 ft., $\$ 2.50$ each.

6-8 ft., $\$ 3.00$ each.

\section{MORUS. Mulberry}

M. tatarica pendula. Tea's Weeping Mulberry. One of the prettiest small weeping trees; forms an umbrella shaped head with slender branches drooping to the ground.

4-5 ft., $\$ 3.00$ each.

\section{PERSICA. Peach}

P. vulgaris alba plena. Double White Flowering Peach. Very hardy and ornamental; a mass of double white flowers.

$$
2 \mathrm{ft} ., \$ 10.00 \text { per } 10 .
$$

3-4 ft., $\$ 12.50$ per 10.

P. Crimson. Double Flowering Crimson Peach. Beautiful dark erimson flowers.

$3-4$ ft., $\$ 12.50$ per 10 .

\section{PJELLODENDRON. Cork Tree}

P. amurense. Chinese Cork Tree. A rapid growing tree, resembling the Ailanthus in habit; thick, corky bark, pinnate foliage, greenish flower.

4- 6 ft., $\$ 2.00$ each.

6 - 8 ft., $\$ 3.00$ each.

8-10 ft., $\$ 4.00$ each. 


\section{PLATANUS. Plane Tree}

P. orientalis. Oriental Plane Tree. A well known tree of rapid growth, valuable on account of its being immune from injurious insects and disease; immense foliage, deeply cut, good for street and park planting.

$$
\begin{array}{r}
8-10 \text { ft., } \$ 20.00 \text { per } 10 \text {. } \\
10-12 \text { ft., } \$ 25.00 \text { per } 10 . \\
12-14 \text { ft., } \$ 45.00 \text { per } 10 .
\end{array}
$$

\section{POPULUS. Poplar}

P. alba. White or Silver Poplar. European tree of very rapid growth and spreading habit. Large, glossy leaves, white on under side.

8-10 ft., $\$ 20.00$ per 10 .

10-12 ft., $\$ 30.00$ per 10.

P. fastigiata. Lombardy Poplar. The well-known pyramidal variety, quick grower, highly effective on a ridge or elevation.

8-10 ft., $\$ 12.00$ per 10 .

10-12 ft., $\$ 20.00$ per 10 .

$12-14$ ft., $\mathrm{X}, \$ 35.00$ per 10.

P. Monilifera. Carolina Poplar. A very quick grower, valuable where immediate effect is desired, or for screens.

8-10 ft., $\$ 10.00$ per 10 .

10-12 ft., $\$ 15.00$ per 10 .

12-14 ft., $\mathrm{X}, \$ 25.00$ per 10 .

\section{PYRUS or Crab Apple}

P. coronaria. Garland Flowered Crab. Single blush flowers.

3-4 ft., $\$ 15.00$ per 10.

4-5 ft., $\$ 20.00$ per 10 .

P. floribunda. Single flowers, carmine in bud, white when open; ornamental fruit.

3-4 ft., $\$ 15.00$ per 10 .

4-5 ft., $\$ 20.00$ per 10 .

P. ioensis. Bechtel's Double Flowering Crab. Large double, fragrant flowers of delicate pink in Spring; graceful tree, very hardy.

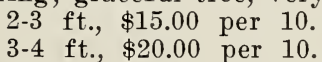

P. Parkmanii. Parkman's Flowering Crab. Long narrow shining green leaves, semi-double carmine flowers in May.

3-4 ft., $\$ 15.00$ per 10 .

4-5 ft., $\$ 20.00$ per 10 .

P. spectabilis. Double Chinese Crab. Fragrant flowers in May, white and rose flowered form. $3-4 \mathrm{ft}$., $\$ 20.00$ per 10 .

\section{QUERCUS. Oak}

Q. alia. White Oak. One of the finest American trees, lobed, pale green above, whitish below.

6- 8 ft., $\$ 30.00$ per 10 .

8-10 ft., $\$ 40.00$ per 10 .

Q. coccinea. Scarlet Oak. Native tree of rapid pyramidal growth, bright red foliage in Autumn.

8-10 ft., $\$ 3.50$ each.

10-12 ft., $\$ 5.00$ each.

$12 \mathrm{ft} ., \$ 7.00$ each. 
QUERCUS-Continued

Q. palustris. Pin Oak. Deep green, finely divided

leaves; excellent for street or lawn planting.

6- $8 \mathrm{ft}$., $\$ 20.00$ per 10 .

8-10 ft., $\$ 30.00$ per 10 .

10-12 ft., $\$ 50.00$ per 10 .

$12-14$ ft., $\$ 7.00$ each.

Q. rubra. Red Oak. Large native tree, hardy and rapid in growth, purplish-red in Autumn. 8-10 ft., $\$ 35.00$ per 10 . $10 \mathrm{ft.}, 1 \frac{1 / 2}{2}$ in. cal., $\$ 40.00$ per 10 .

\section{SALISBURIA \\ Ginkgo or Maiden-Hair Tree}

S. adiantifolia. Ginkgo, or Maidenhair Tree. Japanese tree of distinctive habit, with wedgeshaped leaves like the Maidenhair fern, hardy. 6 - 8 ft., $\$ 20.00$ per 10 . 8-10 ft., $\$ 25.00$ per 10 . 10-12 ft., $\$ 35.00$ per 10 .

\section{SALIX. Willow}

S. babylonica. Weeping Willow. A familiar and graceful tree.

$$
\begin{array}{r}
6-8 \text { ft., } \$ 10.00 \text { per } 10 . \\
8-10 \text { ft., } \$ 15.00 \text { per } 10 . \\
10-12 \text { ft., } \$ 20.00 \text { per } 10 .
\end{array}
$$

s. elegantissima. Thurlow's Weeping Willow. More spreading in habit than Babylonica, branches long and pendant, considered very hardy.

6 - $8 \mathrm{ft} ., \quad \$ 9.00$ per 10.

8-10 ft., $\$ 12.50$ per 10 .

10-12 ft., $\$ 20.00$ per 10 .

s. pentandra. Laurel-Leaved Willow. Large, shining leaves, quick grower, hardy and luxuriant, very useful.

4- 6 ft., $\$ 8.00$ per 10 .

6- 8 ft., $\$ 10.00$ per 10 .

8-10 ft., $\$ 15.00$ per 10.

s. vitellina aurea. Golden Willow. Handsome tree, with yellow bark; very effective in Winter.

4-6 ft., $\$ 9.00$ per 10 .

S. vitellina britzensis. Bark bright red in Winter, very attractive.

4-6 ft., $\$ 9.00$ per 10 .

6-8 ft., $\$ 12.00$ per 10 .

\section{SORBUS}

S. aucuparia. European Mountain Ash. Hardy tree covered from July until Winter with bright scarlet berries.

4- $6 \mathrm{ft}$., $\$ 12.50$ per 10 .

6 - $8 \mathrm{ft}$., $\$ 20.00$ per 10 .

8-10 ft., $\$ 30.00$ per 10 .

10-12 ft., $\$ 40.00$ per 10 .

\section{TILIA. Linden or Lime}

T. americana. American Linden. A fine native tree, with large leaves and creamy yellow, fragrant flowers; rapid grower.

8-10 ft., $\$ 20.00$ per 10 .

10-12 ft., $\$ 35.00$ per 10 .

12 ft., 2 in. cal., $\$ 45.00$ per 10. 


\section{TILIA-Continued}

T. europaea. European Linden. Small leaves; a moderate grower.

8-10 ft., $\$ 25.00$ per 10 .

$10-11$ ft., $\$ 35.00$ per 10 .

11-12 ft., $\$ 45.00$ per 10.

T. europaea argentea. Silver Linden. Pyramidal tree, leaves smooth above and downy beneath, very white in general effect.

8-10 ft., $\$ 4.00$ each.

$10-12$ ft., $\$ 5.00$ each.

$12-14$ ft., $\$ 6.00$ each.

T. europaea platyphylla. Broad-Leaved European Linden. Larger and rougher leaves than Europaea, more regular growth.

$10-12$ ft., $\$ 4.00$ each.

$12-13$ ft., $\$ 6.00$ each.

\section{ULMUS. Elm}

U. americana. American Elm. The finest native tree for street and general planting, noble in growth and assuming many forms.

8-10 ft., $\$ 20.00$ per 10.

$10-12$ ft., $\$ 30.00$ per 10 .

$12-14$ ft., $\$ 40.00$ per 10 .

\section{Evergreens}

\section{ABIES. Fir}

A. concolor. Colorado Silver Fir. Regarded as the finest Rocky Mountain evergreen, foliage bluish above, silvery beneath; habit of tree stately.

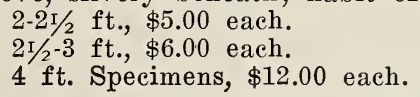

A. Douglasii. Douglas Spruce. Horizontally spreading branches, conical form, light green leaves, glaucous beneath.

$\begin{array}{lll}2 & \text { ft., } \$ 3.00 & \text { each. } \\ 2 \mathrm{r} / 2 & \text { ft., } \$ 4.00 & \text { each. } \\ 3 & \text { ft., } \$ 5.00 & \text { each. } \\ 4 & \text { ft., } \$ 8.00 & \text { each. } \\ 5 & \text { ft., } \$ 9.00 & \text { each. }\end{array}$

A. Nordmanniana. Nordmann's Silver Fir. A noble tree from Crimea; dark green, shining foliage, whitish beneath; very massive, symmetrical form.

$2-2 \mathrm{x} / 2 \mathrm{ft}$., $\$ 5.00$ each.

$2 \mathrm{r} / 2 \mathrm{ft} ., \$ 6.00$ each.

$3-4$ ft., $\$ 7.00$ each.

A. Veitchii. Dark green, lustrous leaves, silvery beneath; very hardy and one of the most beautiful Firs.

$2 \mathrm{t} / 2 \mathrm{ft} ., \$ 5.00$ each.

\section{BIOTA. Arborvitae}

B. orientalis. Chinese Arborvitae. Pyramidal growth, bright green foliage.

2 ft., $\$ 3.00$ each.

3 ft., $\$ 5.00$ each. 


\section{JUNIPERUS. Juniper}

J. canadensis. Common Juniper. Shrub, sometimes becoming a tree; spreading branches, dark green, sometimes glaucous, bluish fruit.

18-24 in., $\$ 3.00$ each.

J. excelsa stricta. A rapid grower.

12 in., $\$ 2.00$ each.

15 in., $\$ 3.00$ each.

J. hibernica. Narrow columnar form, upright branches, deep green, tips of shoots erect.

18 in., $\$ 2.50$ each.

2 ft., $\$ 3.00$ each.

3 ft., $\$ 4.00$ each.

J. Pfitzeriana. A most graceful Evergreen, blue-green foliage, spreading form, usually branching out and as broad as they are high; a fine tree for border planting.

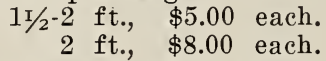

$2 \mathrm{r} / 2$ ft., $\$ 10.00$ each.

J. prostrata. Waukegan Juniper. Long trailing branches, bluish green, blue fruit; very hardy. 18-24 in., $\$ 3.00$ each.

J. Sabina. Savin. Dwarf and trailing; thrives in very poor soil. Useful for rockwork.

15 in., $\$ 3.00$ each.

18 in., $\$ 4.00$ each.

2 ft., $\$ 4.50$ each.

J. suecica. Swedish Juniper. A small, handsome pyramidal tree, yellowish green foliage.

15 -18 in., $\$ 2.00$ each.

$2 \mathrm{ft} ., \$ 3.00$ each.

J. virginiana. Red Cedar. A familiar native tree with dark green foliage and blue berries, makes a good hedge.

2 ft., $\$ 2.50$ each.

3 ft., $\$ 4.00$ each.

\section{PICEA. Spruce}

P. alba. White Spruce. Silvery gray foliage, light colored bark; pyramidal form.

2 ft., $\$ 3.50$ each.

$2 \mathrm{r} / 2$ ft., $\$ 4.00$ each.

P. excelsa. Norway Spruce. Rapid growing pyramidal tree, lively green, branches droop gracefully as the tree attains size. Excellent in masses for windbreaks, hedges, or as single specimens.

$2 \mathrm{ft}$., $\$ 2.00$ each.

$3 \mathrm{ft}$., $\$ 3.00$ each.

4 ft., $\$ 4.00$ each.

P. orientalis. Eastern Spruce. A tall, compact tree with graceful habit and slender foliage.

2 ft., $\$ 5.00$ each.

$3 \mathrm{ft}$., $\$ 8.00$ each.

P. pungens. Colorado Spruce. The original Colorado Blue Spruce. The foliage is sometimes blue, occasionally green, a strong grower, hardy and is a beautiful tree.

3 ft., $\$ 7.00$ each.

4 ft., $\$ 11.00$ each. 


\section{PICEA-Continued}

P. pungens Kosteriana. Koster's Blue Spruce. A very handsome form with bright blue foliage; makes one of the choicest speciments.

$2 \mathrm{ft.}, \$ 12.00$ each.

3 ft., $\$ 18.00$ each.

4 ft., $\$ 24.00$ each.

5 ft., $\$ 30.00$ each.

\section{PINUS. Pine}

P. austriaca. Austrian Pine. Very robust, hardy, and spreading, long, stiff, dark green leaves, rapid grower; good for seashore planting.

3 ft., $\$ 5.00$ each.

4 ft., \$7.50 each.

P. excelsa.. Bhotan Pine. An elegant tree with drooping silvery foliage, of graceful habit and very

vigorous. A native of the Himalayas, and hardy.

$3 \mathrm{ft} ., \$ 4.50$ each.

$4 \mathrm{ft} ., \$ 5.50$ each.

P. Mughus. Dwarf Mugho Pine. A small, compact grower, usually forming a picturesque dwarf bush, dense deep green.

12 in., $\$ 3.00$ each.

18 in., $\$ 4.50$ each.

24 in., \$6.50 each.

P. rigida. Pitch Pine. Horizontal, spreading branches, dark green, strong and rapid grower.

2 ft., $\$ 3.00$ each.

3 ft., $\$ 4.00$ each.

P. Strobus. White, or Weymouth Pine. Light, silvery green foliage, picturesque growth, grows well on poor, sandy soil, very hardy.

2 ft., $\$ 2.00$ each.

3 ft., $\$ 3.50$ each.

4 ft., $\$ 4.50$ each.

P. sylvestris. Scoteh Pine. Robust, rapid grower, erect habit, silvery green foliage, excellent for shelter or windbreak.

2 ft., $\$ 2.00$ each.

$3 \mathrm{ft}$., $\$ 3.50$ each.

$4 \mathrm{ft} ., \$ 5.00$ each.

\section{RETINISPORA. Japan Cypress}

R. filifera. Loosely pyramidal bush or smal ltree; very feathery drooping foliage, dark green.

$$
\begin{aligned}
& 2 \mathrm{ft} ., \$ 4.00 \text { each. } \\
& 2 \mathrm{r} / 2 \mathrm{ft} ., \$ 5.00 \text { each. }
\end{aligned}
$$

R. filifera aurea. A golden form of the preceding, making a very handsome lawn specimen.

$$
\begin{aligned}
& 12 \text { in., } \$ 3.00 \text { each. } \\
& \text { 15-18 in., } \$ 4.50 \text { each. }
\end{aligned}
$$

R. obtusa. Dwarf and rounded, very dense, spreading bright green leaves; grows to a height of $30 \mathrm{ft}$.

15-18 in., $\$ 2.00$ each.

18-24 in., $\$ 3.00$ each.

$2-2 \mathrm{I} / 2$ ft., $\$ 4.50$ each. 


\section{RETINISPORA-Continued}

R. pisifera. Sawara Cypress. Horizontal, flattened branches, somewhat pendulous, bright green, making a large shrub of graceful habit.

18 in., $\$ 3.00$ each.

2 ft., $\$ 4.50$ each.

3 ft., $\$ 6.50$ each.

R. pisifera aurea. Golden Pea-Fruited Japan Cypress. Yellow foliage; a beautiful specimen.

18 in., $\$ 3.00$ each.

2 ft., $\$ 4.00$ each.

3 ft., $\$ 6.50$ each.

R. plumosa. Dense conical habit, slender feathery branchlets; bright green. Can be pruned into a compact specimen, or formed into a broad garden hedge of unusual beauty.

$$
\begin{array}{r}
18 \text { in., } \$ 3.00 \text { each. } \\
2 \text { ft., } \$ 4.00 \text { each. } \\
3 \text { ft., } \$ 6.50 \text { each. }
\end{array}
$$

R. plumosa aurea. Golden Japanese Cypress. Young growths golden yellow, and extremely showy form, making a fine specimen.

$$
\begin{aligned}
& 11 / 2 \mathrm{ft} \text {., } \$ 3.00 \text { each. } \\
& 2 \text { ft., } \$ 4.50 \text { each. } \\
& 2 \mathrm{r} / 2 \mathrm{ft} \text {., } \$ 6.50 \text { each. }
\end{aligned}
$$

R. squarrosa Veitchii. Exceedingly dense and bushy with spreading, feathery branches; steel blue, very handsome.

$$
\begin{array}{ccc}
18 \text { in., } \$ 3.50 \text { each. } \\
2 \text { ft., } \$ 4.50 \text { each. } \\
21 / 2 \text { ft., } \$ 6.00 \text { each. }
\end{array}
$$

\section{TAXUS. Yew}

T. baccata. English Yew. Large, bushy tree; very dense, dark green, ean be trimmed in any shape.

$$
12-15 \text { in., } \$ 2.50 \text { each. }
$$

15-18 in., \$3.50 each.

T. brevifolia. Japanese Yew. Leaves short and dark green. A handsome tree.

$$
12 \text { in., } \$ 3.00 \text { each. }
$$

15 in., $\$ 4.00$ each.

T. canadensis. Canadian Yew. Low spreading and bushy dark green foliage and bright red berries. Hardy.

12 in., $\$ 2.50$ each.

T. cuspidata. Japanese Yew. Dense habit, dark green foliage, lighter beneath, branches ascending; hardiest of Yews.

15 in., $\$ 3.00$ each.

18 in., $\$ 4.00$ each.

2 ft., $\$ 5.00$ each.

\section{THUYA. Arborvitae}

T. compacta. Dwarf and compact, with deep green foliage.

12 in., $\$ 2.00$ each.

18 in., $\$ 3.00$ each.

T. globosa. Dwarf, rounded form, bright green; handsome.

12-15 in., $\$ 2.00$ each.

15-18 in., $\$ 2.50$ each.

18-24 in., $\$ 4.00$ each. 


\section{THUYA-Continued}

T. Hoveyi. Dwarf, dense, rounded oval form, light green shading to yellow on young growth.

12 in., $\$ 2.00$ each.

18 in., $\$ 3.00$ each.

T. occidentalis. Common Arborvitae, or White Cedar. Short, horizontal branches forming a narrow pyramid, bright green, very hardy, rapid grower, submits to close shearing and endures any soil.

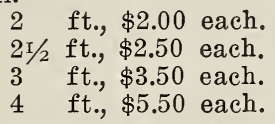

T. occidentalis pyramidalis. Pyramidal Arborvitae. A pyramidal form of the Asiatic Arborvitae. Bright green foliage; one of the tallest and hardiest varieties.

$$
\begin{array}{llll}
2 & \text { ft., } \$ 2.50 & \text { each. } \\
2 \mathrm{I} / 2 & \mathrm{ft.} & \$ 3.00 & \text { each. } \\
3 & \text { ft., } & \$ 4.00 & \text { each. } \\
4 & \text { ft., } & \$ 5.00 & \text { each. }
\end{array}
$$

T. occidentalis siberica. Siberian Arbovitae. Pyramidal habit, rich green, keeping color well through Winter. Excellent for screens and windbreaks.

18 in., $\$ 2.25$ each.

2 ft., $\$ 3.00$ each.

3 ft., $\$ 5.00$ each.

\section{TSUGA. Hemlock}

T. canadensis. Common Hemlock, or Hemlock Spruce. A rapid grower and hardy, which can be sheared into a bushy form; makes a fine hedge. Good for seashore planting.
2 ft., $\$ 3.50$ each.
3 ft., $\$ 5.00$ each.
4 ft., $\$ 7.00$ each.
5 ft., $\$ 8.00$ each.

\section{Evergreen Shrubs}

\section{ANDROMEDA}

A. Catesbaei. Long, recurving branches, handsome pointed leaves, turning bronze in Winter, frarrant white flowers in Spring.

$12-15$ in., $\$ 2.00$ each.

18 in., $\$ 3.00$ each.

A. floribunda. Pointed leaves, thickly set; abundan white flowers, suggestive of the Lily of the Val ley in early Spring.

$12-15$ in., $\$ 35.00$ per̀ 10.

15-18 in., \$45.00 per 10.

\section{AZALEA}

A. amoena. A Chinese Azalea; very dwarf an bushy, covered with dark red flowers in Ma? abundant small leaves, persisting all Winte turning reddish brown in Autumn.

10-12 in., $\$ 2.00$ each.

12-15 in., $\$ 3.00$ each. 


\section{AZALEA-Continued}

A. amoena var. Hinodigiri. Resembles Amoena in growth, flowers are larger and more brilliant, color a bright red or carmine-pink.

8-10 in., $\$ 2.00$ each.

10-12 in., $\$ 3.00$ each.

12-15 in., \$4.00 each.

\section{BUXUS. BoX}

B. suffruticosa. Dwarf Box. A dwarf form with small, oval leaves, good for edging.

3 in., $\$ 12.00$ per 100 .

4 in., $\$ 16.00$ per 100 .

6 in., $\$ 20.00$ per 100 .

\section{COTONEASTER}

C. horizontalis. Small-leaved Cotoneaster. Trailing shrub with small leaves, white flowers followed by red berries.

12 in., $\$ 10.00$ per 10.

C. microphylla.

$1 \mathrm{I} / 2$ ft., $\$ 15.00$ per 10 .

$\begin{array}{lll}12 & \text { in., } \$ 11.00 \text { per } 10 . \\ 15 & \text { in., } \$ 13.00 \text { per } 10 . \\ 18 \text { in., } \$ 15.00 \text { per } 10 .\end{array}$

DAPHNE

D. Cneorum. Trailing Daphne. Dwarf trailing plant, with evergreen leaves and fragrant, pink or white flowers in early Spring.

6 - 8 in., $\$ 12.00$ per 10.

8-10 in., $\$ 17.00$ per 10 .

\section{EUONYMUS}

E. japonicus. Upright shrub with narrow evergreen leaves, pinkish fruit.

9-12 in., $\$ 6.00$ per 10.

$12-15$ in., $\$ 7.50$ per 10 .

\section{ILEX. Holly}

I. crenata. Japanese Holly. Shining leaves, waxy at the edge, much hardier than the English Holly, very desirable for hedges, and also for lawn specimens.

$12-18$ in., $\$ 2.00$ each.

18-24 in., $\$ 3.00$ each.

\section{KALMIA. Laurel Nursery Grown}

K. latifolia. Among the most beautiful of all flowering evergreens, is the American Laurel. The foliage is very decorative, the ordinary Kalmia latifolia being almost covered with its showy, flesh-pink flowers. The Kalmia requires the same conditions as the Rhododendrons.

15 in., $\$ 2.50$ each.

$1 \mathrm{1} / 2 \mathrm{ft}$., $\$ 3.00$ each.

2 ft., $\$ 4.00$ each.

Collected plants in car lot. Write for price. 


\section{RHODODENDRONS}

R. maximum. Both nursery grown and collected plants of white flowers. 1-6 ft.

Send list of wants for price.

R. carolinianum. Pink flower, plants 1 to $4 \mathrm{ft}$.

Write for price.

R. catawbiense. Color of flower a deep pink slightly tinted with lavender, flower of immense size and perfectly hardy, collected plants from 1 to $4 \mathrm{ft}$. Write for price.

\section{Deciduous Shrubs}

\section{ABELIA}

A. grandiflora. A beautiful shrub with pink flowers.

Leaves turn a deep red in Fall.

$$
\begin{aligned}
& 18-24 \text { in., } \$ 12.50 \text { per } 10 ; \$ 100.00 \text { per } 100 . \\
& 2-3 \text { ft., } \$ 20.00 \text { per } 10 ; \$ 150.00 \text { per } 100 . \\
& 3-4 \text { ft., } \$ 25.00 \text { per } 10 ; \$ 200.00 \text { per } 100 .
\end{aligned}
$$

\section{AMORPHA. False Indigo}

A. fruiticosa. False Indigo. Hairy shrub, dense pan-

icles of dark purple flowers in June and July. $2-3$ ft., $\$ 5.50$ per 10.

3-4 ft., $\$ 7.00$ per 10 .

\section{AMYGDALUS. Almond}

A. Double White and Pink Flowering Almond. Long drooping branches of beautiful double white and pink flowers in May.

2-3 ft., $\$ 10.00$ per 10.

3-4 ft., $\$ 12.50$ per 10 .

\section{ARALIA. Angelica Tree}

A. Pentaphylla. Angelica Tree. Pretty shrub with five-lobed pale green leaves; spiny.

2 ft., $\$ 5.00$ per $10 ; \$ 40.00$ per 100 .

3 ft., $\$ 8.00$ per $10 ; \$ 70.00$ per 100 .

\section{AZALEA}

A. arborescens. Large and spreading handsome dark green foliage and white or rosy blossoms late in Summer.

$15-18$ in., $\$ 25.00$ per 10 .

$18-24$ in., $\$ 30.00$ per 10 .

$2-21 / 2$ ft., $\$ 36.00$ per 10.

A. calendulacea. Yellow and flame colored flowers late in May, very showy.

12-18 in., $\$ 15.00$ per 10 .

18-24 in., $\$ 20.00$ per 10.

2 ft., $\$ 36.00$ per 10.

A. mollis. Chinese Azalea. Largely of orange color. 12 in., $\$ 3.00$ each. 15-18 in., \$4.00 each.

A. nudifiora. Pinxter Flower. Beautiful native shrub with rose and purplish pink flowers.

$1-2$ ft., $\$ 7.00$ per 10 .

2-4 ft., \$9.00 per 10 .

A. Vaseyi. White and pink.

15-18 in., $\$ 26.00$ per 10. 


\section{BACCHARIS. Groundsel Tree}

B. halimifolia. Groundsel Tree. Dark green foliage, fluffy white seed pods in Autumn, fine for seaside planting.

$\begin{array}{llll}2-3 & \text { ft., } \$ 7.00 & \text { per } 10 . \\ 3-4 \text { ft., } \$ 10.00 \text { per } 10 .\end{array}$

\section{BENZOIN. Wild Allspice}

B. odoriferum. Wild Allspice. Yellow flowers in Spring, oval leaves, bright red berries, persisting through Winter.

$\begin{array}{llll}2-21 / 2 & \text { ft., } & \$ 7.00 & \text { per } 10 . \\ 3-4 & \text { ft., } & \$ 9.00 & \text { per } 10 .\end{array}$

\section{BERBERIS. Barberry}

B. Thunbergii. Thunberg's Barberry, Dwarf, compact growth, small foliage, turning a rich red in

Autumn. Valuable as a hedge plant.

$18-24$ in., $\$ 6.00$ per $10 ; \$ 50.00$ per 100 .

$2-3 \mathrm{I} / 2$ ft., $\$ 9.00$ per $10 ; \$ 60.00$ per 100 .

B. vulgaris purpurea. Purple-leaved Barberry. Foliage and fruit violet purple, very handsome.

$18-24$ in., $\$ 5.00$ per $10 ; \$ 35.00$ per 100 .

2 - 3 ft., $\$ 6.00$ per $10 ; \$ 40.00$ per 100 .

\section{BUDDLEIA}

B. intermedia. Violet-colored flowers in slender arching, pendu ous racemes.

2 Yr., $\$ 10.00$ per 10.

B. Veitchii. An improved variety, similar in its growth, but has finer flowers, larger spikes, more dense and brilliant violet in color, with orangeyellow centre.

18-24 in., $\$ 6.00$ per 10.

2- 3 ft., $\$ 7.00$ per 10 .

\section{CALLICARPA. French Mulberry}

C. purpurea. Purple Callicarpa. Dwarf shrub, with small, purple flowers in August and September. $11 \frac{1}{2}-2$ ft., $\$ 5.00$ per 10 . $2-21 / 2$ ft., $\$ 6.00$ per 10.

\section{CALYCANTHUS. Carolina Allspice}

C. floridus. Carolina Allspice. Sweet-scented shrub. Chocolate-colored flowers in May, glossy; dark leaves. Both flowers and twigs have a rich, aromatic fragrance, very hardy and desirable.

$2-3 \mathrm{ft}$., $\$ 7.00$ per 10 .

3-4 ft., $\$ 9.00$ per 10 .

\section{CARYOPTERIS. Blue Spirea}

C. Mastacanthus. Low shrub, producing profuse clusters of fragrant blue flowers from September to frost, very handsome. 15-18 in., $\$ 4.00$ per 10. 18 in., $\$ 5.00$ per 10.

\section{CEANOTHUS. New Jersey Tea}

C. americana. New Jersey Tea, or Red Root. Dwarf shrub bearing white flowers profusely in June; does well in shade.

18-24 in., $\$ 7.00$ per 10 .

2- 3 ft., $\$ 9.00$ per 10. 


\section{CHIONANTHUS. White Fringe}

c. virginica. White Fringe. Large, dark green leaves, long racemes of fringe-like flowers in May; purple fruit in the Fall, very desirable.

2-3 ft., $\$ 12.00$ per 10 .

3-4 ft., $\$ 15.00$ per 10 .

\section{CLETHRA. Sweet Pepper Bush}

C. alnifolia. Native shrub with small spikes of fragrant white flowers in July, low, dense growth.

$2-21 / 2$ ft., $\$ 6.00$ per 10 .

3-4 ft., $\$ 8.00$ per 10 .

\section{COLUTEA. Bladder Senna}

C. arborescens. Tall shrub with fine Acacia-like leaves, yellow flowers in June, followed by round, reddish pods.

2-3 ft., $\$ 6.00$ per 10.

3-4 ft., $\$ 7.00$ per 10 .

\section{CORNUS. DOGWOOD}

c. alba. Red-Twigged Dogwood. White flowers in June; bright red bark, making it conspicuous in Winter.
2-3 ft., $\$ 5.00$ per 10 .

3-4 ft., $\$ 6.00$ per 10 .

c. elegantissima. Pale yellow flowers.

2-3 ft., $\$ 8.00$ per 10 .

3-4 ft., $\$ 9.00$ per 10 .

C. Iutea. Golden Margined Dogwood. Leaves bordered with pale yellow, some entirely yellow.

$2-3$ ft., $\$ 5.00$ per 10 .

3-4 ft., $\$ 6.00$ per 10 .

C. mascula. Cornelian Cherry. Clusters of bright yellow flowers in Spring before the leaves, followed by red berries; shining leaves, dense habit.

$2-3$ ft., $\$ 5.00$ per 10 .

$3-4$ ft., $\$ 6.00$ per 10 .

4-5 ft., $\$ 9.00$ per 10 .

c. paniculata. Panicled Dogwood. Smooth bark, pointed white green leaves, whitish beneath greenish white flowers, white fruit.

$2-3$ ft., $\$ 5.00$ per 10 .

3-4 ft., $\$ 6.00$ per 10 .

C. stolonifera. Wild Red Osier Dogwood. Native species of spreading habit, smooth slender branches, dark red in Winter.

3-4 ft., $\$ 5.00$ per 10 .

4-5 ft., $\$ 9.00$ per 10 .

\section{CORYLUS. Hazel}

C. Avellana purpurea. Purple-Leaved Filbert. With large, dark purple leaves.

18 in., $\$ 10.00$ per 10 .

\section{CYDONIA. Japanese Quince}

C. japonica. Japan Quince. Brilliant erimson flowers; neat foliage; makes a fine hedge.

18 in., $\$ 6.00$ per 10 .

2-3 ft., $\$ 8.00$ per 10 . 


\section{DESMODIUM}

D. penduliflorum. Low growing shrub with rosepurple flowers in September, dies down in Winter.

2 Yrs., $\$ 6.00$ per 10.

\section{DEUTZIA}

D. candidissima. A pure white form, free bloomer.

$$
\begin{aligned}
& \text { 2-3 ft., } \$ 5.00 \text { per } 10 \text {. } \\
& \text { 3-4 ft., \$6.00 per } 10 \text {. }
\end{aligned}
$$

D. crenata. Double and single pink flowers.

$2-3$ ft., $\$ 5.00$ per 10 .

3.4 ft., $\$ 6.00$ per 10.

D. gracilis. Dwarf shrub, a mass of graceful white flowers, about the middle of June, before the other Deutzias.

$$
\text { 18-24 in., } \$ 6.00 \text { per } 10 .
$$

D. Pride of Rochester. A hybrid of Crenata, double white tinged rose, long, graceful panicles. Blooms earlier than Crenata.

$2-3$ ft., $\$ 4.00$ per 10 .

3-4 ft., $\$ 6.00$ per 10 .

D. Lemoinei. A hybrid of Gracilis, dwarf and free flowering, single.

$$
2-3 \text { ft., } \$ 5.00 \text { per } 10 \text {. }
$$$$
\text { 3-4 ft., } \$ 7.00 \text { per } 10 \text {. }
$$

\section{DIERVILLA. Weigela}

D. candida. White Flowered Weigela. Creamy white flowers, freely produced in June; strong grower. $2-3$ ft., $\$ 6.00$ per 10 .

3-4 ft., $\$ 7.00$ per 10 .

D. Desboisii. Fine shrub with deep, rose-colored flowers, very good. 2 -3 ft., $\$ 7.00$ per 10 .

D. Eva Rathke. Flowers brilliant crimson, distinct from all others, a new variety.

$\begin{array}{lll}2 & \text { ft., } \$ 8.00 & \text { per } 10 . \\ 21 / 2 & \text { ft., } \$ 9.00 & \text { per } 10 .\end{array}$

D. nana follis variegata. Dwarf Variegated Weigela. Dwarf habit, leaves with silvery variegation, flowers nearly white.

2-3 ft., $\$ 7.00$ per 10 .

3-4 ft., $\$ 9.00$ per 10 .

D. rosea. Rose Colored Weigela. Erect growth, abundant rose colored flowers in June.

$$
\begin{array}{llll}
2-3 & \text { ft., } & \$ 6.00 & \text { per } 10 . \\
3-4 & \text { ft., } & \$ 8.00 & \text { per } 10 .
\end{array}
$$

\section{ELAEAGNUS. Silver Thorn}

E. augustifolia. Shrub or small tree of graceful habit, small yellow flowers, followed by oval silvery, yellow fruit.

2-3 ft., $\$ 7.00$ per 10 .

3-4 ft., $\$ 8.00$ per 10 .

E. Longipes.. Oleaster. Thin, oval leaves, long drooping branches, small flowers, followed by drooping, oval fruit, which makes an excellent jelly.

2-3 ft., $\$ 6.00$ per 10 .

$3-4$ ft., $\$ 9.00$ per 10 . 


\section{EUONYMUS}

\section{SPINDLE TREE or Burning Bush}

E. alatus. Cork Barked Spindle Tree. Dwarf habit, corky bark, small leaves turning bright red in Autumn, red fruit.

2-3 ft., $\$ 9.00$ per 10 .

3-4 ft., $\$ 12.00$ per 10.

E. americana. Strawberry Tree. Showy red berries. $2 \mathrm{ft} ., \$ 6.00$ per 10 .

E. europaeus. European Spindle Tree. Large shrub or small tree, showy rose colored fruit, in $\mathrm{Au}$ tumn foliage reddens.

2-3 ft., $\$ 6.00$ per 10 .

\section{EXOCHORDA. Pearl Bush}

E. grandiflora. Pearl Bush. Beautiful shrub with light green leaves and large sprays of white flowers in May.

$2-3$ ft., $\$ 6.00$ per 10 .

$3-4$ ft., $\$ 8.00$ per 10 .

\section{FORSYTHIA. Golden Bell}

F. Fortunei. Golden Bell. Upright growth, deep green leaves, showy yellow flowers in Spring.

2-3 ft., $\$ 4.00$ per 10.

$3-4$ ft., $\$ 5.00$ per 10.

F. intermedia. Very similar to suspensa, hardy and very floriferous.

2-3 ft., $\$ 4.00$ per 10.

$3-4$ ft., $\$ 6.00$ per 10.

F. suspensa. Weeping Golden Bell. Similar to Fortunei, but with drooping branches, profuse yellow flowers.

2-3 ft., $\$ 5.00$ per 10 .

$3-4$ ft., $\$ 6.00$ per 10.

F. viridissima. Leaves and bark deep green, abundant yellow flowers early in Spring, very hardy.

2-3 ft., \$4.00. per 10 .

3 -4 ft., $\$ 6.00$ per 10 .

\section{HALESIA. Snowdrop Tree}

H. tetraptera. Four-winged Snowdrop Tree. Large shrub with bell-shaped white flowers in May. $2-3$ ft., $\$ 8.00$ per 10 .

\section{HAMAMELIS. Witch Hazel}

H. virginica. Witch Hazel. Tall shrub with yellow flowers in Autumn, just before the leaves fall. $2-3$ ft., $\$ 6.00$ per 10 .

$3-4$ ft., $\$ 8.00$ per 10.

\section{HIBISCUS}

\section{Althaea or Rose of Sharon}

H. syriacus. Althaea or Rose of Sharon. These handsome shrubs bloom in August and September, when other trees and shrubs are out of bloom. Very hardy and easily grown. Named varieties. 2-3 ft., $\$ 4.00$ per 10 . $3-4$ ft., $\$ 5.00$ per 10 . 


\section{HYDRANGEA}

H. arborescens sterilis forma nova. Snowball Hydrangea, or Hills of Snow. (New). Snowball white blossoms of the largest size, lacking the coarseness of $\mathrm{H}$. paniculata grandiflora, form of panicle is similar to Hortensis. Most desirable on account of bloom after the early Spring shrubs, blooming from early June through August.

2-3 ft., $\$ 8.00$ per 10.

3-4 ft., $\$ 9.00$ per 10 .

H. otaksa.. Japanese Hydrangea. Large leaves, immense heads of blue or pink flowers.

$12-15$ in., $\$ 10.00$ per 10 .

H. paniculata, Hardy Hydrangea. Elongated panicles of white single flowers in Summer, large leaves.

$2-3$ ft., $\$ 7.00$ per 10 .

3-4 ft., $\$ 8.00$ per 10 .

H. paniculata grandiflora. Large panicled Hydrangea. One of the showiest of Summer blooming shrubs, huge elongated panicles of white flowers, turning rose-colored with age.
$2-3$ ft., $\$ 8.00$ per 10.

$3-4$ ft., $\$ 10.00$ per 10.

H. New French Varieties. In assorted varieties and colors of rose, white and pink.

$12-15$ in., $\$ 15.00$ per 10.

$15-18$ in., $\$ 20.00$ per 10.

\section{HYPERICUM. St. John's Wort}

H. aureum. Golden St. John's Wort. Upright in growth, with bright yellow flowers from July to October, very fine.

$11 / 2-2$ ft., $\$ 5.00$ per 10.

$2-21 / 2$ ft., $\$ 6.00$ per 10.

\section{ILEX. Mountain Holly. Alder}

I. verticillata. Black Alder. Glossy dark green leaves, white flowers, bright red berries in Fall.

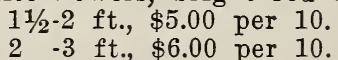

\section{ITEA. Virginia Tea}

I. virginica. Virginia Tea. Native shrub with pretty white flowers in June, foliage rich deep red in Fall.

$11 / 2-2$ ft., $\$ 6.00$ per 10.

2 -3 ft., $\$ 7.00$ per 10 .

\section{LIGUSTRUM. Privet}

L. amurense. Amoor Privet. Compact in growth, small leaves, almost evergreen, does well in a dry place.

$$
\begin{array}{ll}
2-3 & \text { ft., } \$ 22.00 \text { per } 100 . \\
3-4 \text { ft., } \$ 32.00 \text { per } 100 .
\end{array}
$$

L. ibota. Chinese Privet. Hardier than ovalifolium, large white flowers profusely produced, long, shining leaves; one of the best.

$2-3$ ft., $\$ 24.00$ per 100 .

3.4 ft., $\$ 32.00$ per 100 . 


\section{LIGUSTRUM-Continued}

L. ibota Regelianum. Low, dense shrub, branches spread horizontally, obvate leaves, very hardy. $2-21 / 2 \mathrm{ft}$., $\$ 50.00$ per 100 .

$21 / 2^{-3}$ ft., $\$ 60.00$ per 100 .

L. ovalifolium. California Privet. Vigirous and hardy, bears any amount of shearing; our best shrub for garden hedges.

$18-24$ in., $\$ 7.00$ per 100 .

$2 \mathrm{ft.}, \$ 10.00$ per 100 .

3- 4 ft., $\$ 15.00$ per 100 .

L. ovalifolium aureum. A rare yellow-leaved variety, very desirable.

12-15 in., $\$ 40.00$ per 100.

$15-18$ in., $\$ 60.00$ per 100 .

L. vulgare. Common European Privet. Narrow foliage, showy white flowers.

2-3 ft., $\$ 16.00$ per 100 .

\section{LILAC. (See Syringa) LONICERA. Honeysuckle}

L. Alberti. An upright Honeysuckle. Violet, bellshaped flowers, narrow leaves.

$1 \mathrm{ft} ., \$ 5.00$ per 10 .

L. fragrantissima. Fragrant Upright Honeysuckle. A spreading bush with deep green leaves, almost evergreen, small white, very fragrant flowers before the leaves.

$2-3$ ft., $\$ 6.00$ per 10 .

3-4 ft., $\$ 7.00$ per 10 .

L. Morrowii. A Japanese variety with showy red fruit.

$2-3$ ft., $\$ 6.00$ per 10 .

3-4 ft., $\$ 7.00$ per 10 .

L. tatarica. White and Pink Bush Honeysuckle.

$2-3$ ft., $\$ 5.00$ per $10 ; \$ 40.00$ per 100 .

$3-4$ ft., $\$ 6.00$ per $10 ; \$ 50.00$ per 100 .

\section{MYRICA. Bayberry}

M. cerifera. Bayberry. A native shrub with shining stiff, long, oval leaves, and small, bony fruit, very aromatic; fine for seaside planting.

$11 / 2-2$ ft., $\$ 6.00$ per $10 ; \$ 50.00$ per 100 . $2-3$ ft., $\$ 7.00$ per 10 .

\section{POTENTILLA. Shrubby Cinquefoil}

P. fruiticosa. Spreading shrub of medium size, with starry yellow flowers produced throughout the Summer.

18-24 in., $\$ 6.00$ per 10.

2- 3 ft., $\$ 7.00$ per 10 .

\section{PHILADELPHUS}

\section{Syringa or Mock Orange}

P. coronarius. Common Mock Orange. A familiar favorite with fragrant white flowers.

2-3 ft., $\$ 5.00$ per 10.

3.4 ft., $\$ 7.00$ per 10 . 
PHILADELPHUS-Continued

P. grandiflorus. Large flowered Syringa. Strong grower, flowering late, less fragrant than the common type.

$$
\begin{array}{lllll}
2-3 & \text { ft., } & \$ 5.00 & \text { per } & 10 . \\
3-4 & \text { ft., } & \$ 6.00 & \text { per } & 10 .
\end{array}
$$

P. Lemoinei. Lemoine's Syringa. Large, creamy flowers, freely produced, upright growth.

$2-3$ ft., $\$ 6.00$ per 10 .

\section{PRUNUS. Plum}

P. maritima. Beach Plum. Spreading bush, fine for seaside planting, a mass of snow-white flowers followed by crimson purple fruit, which is edible. 2-3 ft., $\$ 7.00$ per $10 ; \$ 65.00$ per 100 . $3-4$ ft., $\$ 8.00$ per 10 .

P. Pissardii. Purple-Leaved Plum. White flowers in April, handsome purple foliage, retaining its color through the season; very fine.

3-4 ft., $\$ 10.00$ per 10 .

4-5 ft., \$12.00 per 10 .

P. triloba. Flowering Almond. A mass of beautiful large pink flowers in May before the leaves appear; very showy.

$2-3$ ft., $\$ 10.00$ per 10 .

$3-4$ ft., $\$ 12.50$ per 10 .

\section{RHAMNUS. Buckthorn}

R. cathartica. Pürging Buckthorn. Robust, thorny, European shrub with dark green leaves, white flowers and black fruit, fine for hedges.

2-3 ft., $\$ 4.00$ per 10 .

3-4 ft., $\$ 5.00$ per 10 .

\section{RHODOTYPOS. White Kerria}

R. kerrioides. White Kerria. Handsome foliage and large single white flowers, like single Roses in the latter part of May, followed by black, beadlike fruit.

$2-3$ ft., $\$ 6.00$ per 10 .

3 -4 ft., $\$ 8.00$ per 10 .

\section{RHUS. Sumach}

R. aromatica. Fragrant Sumach. Native variety with aromatic, fragrant, greenish-white flowers.

$2-3$ ft., $\$ 6.00$ per 10 .

3-4 ft., $\$ 7.50$ per 10 .

R. copallina. Shining Sumach. Handsome, shining foliage, turning to gorgeous crimson in Autumn, greenish flowers in August.

$2-3$ ft., $\$ 6.50$ per 10 .

3 -4 ft., $\$ 8.00$ per 10 .

R. cotinus. Purple Fringe, or Smoke Tree. Spreading shrub, growing 10 to 12 feet high, covered in mid-Summer with curious, smoke-like, fringy, purplish flowers, very attractive.

2-3 ft., $\$ 9.00$ per 10 .

3-4 ft., $\$ 12.00$ per 10 .

R. glabra. Smooth Sumach. Brilliant crimson foliage, topped with prominent clusters of showy seeds in Autumn.

$2-3$ ft., $\$ 4.50$ per 10 .

$3-4$ ft., $\$ 6.00$ per 10 . 


\section{RHUS-Continued}

R. glabra var. laciniata. Cut-Leaved Sumach. Deeply cut Fern-like leaves, shining green above, whitish below, glowing red in Autumn.

2-3 ft., $\$ 10.00$ per 10 .

R. typhina. Staghorn Sumach. Tall variety with scarlet fruit, foliage a brilliant red in Autumn.

$2-3$ ft., $\$ 4.50$ per 10 .

3-4 ft., $\$ 5.50$ per 10 .

\section{RUBUS. Bramble}

R. odoratus. Flowering Raspberry. Pretty pinkish purple flowers, large leaves.

$2-3$ ft., $\$ 5.00$ per 10 .

3-4 ft., $\$ 6.00$ per 10.

\section{SAMBUCUS. Elder}

S. canadensis. Common Elder. Rapid growing, native shrub, broad heads of white flowers in June, followed by dark red berries.

2 -3 ft., $\$ 5.00$ per 10 .

$3-4$ ft., $\$ 6.00$ per 10 .

S. nigra aurea. Golden Elder. Bright golden yellow foliage, retaining the color throughout the season; very effective among shrubbery.

$2-3 \mathrm{ft} ., \quad \$ 6.00$ per 10 .

3.4 ft., $\$ 7.00$ per 10 .

\section{SPIRAEA}

S. Billardii. Tall growing shrub with pinkish flowers and bright, green leaves.

2-3 ft., $\$ 5.00$ per 10 .

3-4 ft., $\$ 6.00$ per 10 .

S. Bumalda. Dwarf habit, narrow leaves, pink flowers nearly all Summer.

$18-24$ in., $\$ 6.00$ per 10 .

2- 3 ft., $\$ 7.00$ per 10 .

S. callosa alba. Fortune's Dwarf White Spirea. White flowers, blooming all Summer, dwarf and bushy in habit.

18 in., $\$ 6.00$ per 10 .

S. opulifolia. Ninebark. Strong growing native shrub, with white flowers.

$2-3$ ft., $\$ 4.00$ per 10 .

3-4 ft., $\$ 5.00$ per 10 .

S. opulifolia aurea. Golden Ninebark. A variety with golden foliage, very noticeable.

2-3 ft., $\$ 4.00$ per 10 .

3-4 ft., $\$ 5.00$ per 10 .

S. prunifolia fl. pl. Bridal Wreath. Branches covered with pure white flowers in May, very showy and handsome.

$2-3$ ft., $\$ 7.00$ per 10 .

3-4 ft., $\$ 8.00$ per 10.

S. Reevesii fl. pl. Reeve's Spirea. Covered with large, round clusters of double white flowers in June, very showy.

$$
\begin{aligned}
& 2-3 \text { ft., } \$ 5.00 \text { per } 10 \text {. } \\
& \text { 3-4 ft., } \$ 6.00 \text { per } 10 \text {. }
\end{aligned}
$$




\section{SPIREA-Continued}

S. Thunbergii. Thunberg's Spirea. Dwarf, rounded form, white flowers. One of the first to bloom, very satisfactory.

$$
\begin{aligned}
& 2-3 \text { ft., } \$ 7.00 \text { per } 10 \text {. } \\
& \text { 3-4 ft., } \$ 8.00 \text { per } 10 \text {. }
\end{aligned}
$$

S. tomentosa. Hardhack or Steeplebush. Small leaves, rose-purple flowers in a dense terminal panicle.

$2-3$ ft., $\$ 5.00$ per 10 .

3.4 ft., $\$ 7.00$ per 10.

S. VanHouttei. VanHoutte's Spiraea. The finest of all Spiraeas, a mass of large white flowers in May and early June.

$2-3$ ft., $\$ 5.00$ per 10 .

3-4 ft., $\$ 6.00$ per 10 .

\section{STEPHANANDRA}

S. flexuosa. Slender, graceful shrub, with finely eut foliage, loose clusters of white flowers in June.

$$
\begin{array}{lll}
2-3 & \text { ft., } \$ 5.00 & \text { per } 10 \\
3-4 & \text { ft., } \$ 7.00 & \text { per } 10 .
\end{array}
$$

\section{SYMPHORICARPOS Snowberry, Coral Berry}

S. racemosus. Snowberry. Slender branched shrub; pink flowers, followed by waxen white berries lasting into Winter.

$$
\begin{aligned}
& 2-3 \text { ft., } \$ 5.00 \text { per } 10 \text {. } \\
& \text { 3-4 ft., } \$ 7.00 \text { per } 10 \text {. }
\end{aligned}
$$

S. vulgaris. Coral Berry. Compact bush with small bright red berries, lasting into Winter.

$2-3$ ft., $\$ 5.00$ per 10 .

3-4 ft., $\$ 6.00$ per 10 .

\section{STYRAX}

s. japonica. Handsome small tree, with waxy white, bell-shaped flowers gracefully pendant.

3-4 ft., $\$ 9.00$ per 10.

4-5 ft., $\$ 10.00$ per 10.

\section{SYRINGA. Lilac}

S. Charles Tenth. Strong grower with large, loose trusses of reddish-purple flowers.

$2-3$ ft., $\$ 8.00$ per 10 .

3-4 ft., $\$ 10.00$ per 10 .

s. japonica. Japanese Lilac. Becomes a good sized tree with leathery, dark green leaves, great panicles of creamy flowers a month later than other Lilaes.

$\begin{array}{lllll}2-3 & \text { ft., } & \$ 7.00 & \text { per } 10 . \\ 3-4 & \text { ft., } \$ 9.00 & \text { per } 10 .\end{array}$

S. Josikaea. Flowers dark lilac early in June.

$2-3$ ft., $\$ 7.00$ per 10 .

3-4 ft., $\$ 9.00$ per 10 .

S. Lemoinei fl. pl. A choice variety, producing long racemes of double purple flowers and lasting longer than most varieties.

2-3 ft., $\$ 10.00$ per 10.

3-4 ft., $\$ 12.00$ per 10 . 
SYRINGA-Continued

S. Mme. Casimir Perier. Semi-double, pure white. 2-3 ft., $\$ 9.00$ per 10 .

s. Marie Legraye. The finest white Lilac, large panicles of snowy flowers, much used for forcing. $2-3$ ft., $\$ 9.00$ per 10 .

3-4 ft., $\$ 12.00$ per 10 .

s. persica. Persian Lilac. Small foliage and bright purple flowers.

$$
\begin{array}{llll}
2-3 & \text { ft., } \$ 8.00 & \text { per } 10 . \\
3-4 & \text { ft., } \$ 9.00 & \text { per } 10 .
\end{array}
$$

s. persica alba. Persian White Lilac. Creamy white flowers, shaded purple.

2 ft., $\$ 9.00$ per 10 .

3-4 ft., $\$ 10.00$ per 10.

S. rothomagensis. Chinese Lilac. Very large panicles of reddish flowers, abundantly produced.

$2-3$ ft., $\$ 7.00$ per 10.

S. rubra de Marley. Deep reddish purple; one of the best dark kind.

$2-3$ ft., $\$ 7.50$ per 10 .

3-4 ft., $\$ 10.00$ per 10 .

S. Souv. de Louis Spaeth. Immense trusses of deep rosy purple blooms.

2-3 ft., $\$ 8.00$ per 10 .

3-4 ft., $\$ 10.00$ per 10.

S. villosa. Japanese species, with large branching panicles of white flowers, purple in bud, fragrant; two weeks later than other lilacs.

$2-3$ ft., $\$ 7.00$ per 10 .

3-4 ft., $\$ 9.00$ per 10 .

s. vulgaris. Common Lilac. Bluish-purple flowers, always satisfactory.

2-3 ft., $\$ 5.00$ per 10 .

3-4 ft., $\$ 7.00$ per 10 .

S. vulgaris alba. Common White Lilac. A robust grower, bearing abundant white flowers.

2-3 ft., $\$ 7.00$ per 10 .

3-4 ft., $\$ 8.00$ per 10.

S. Pres. Grevy. Large, double blue, very beautiful. 2-3 ft., $\$ 8.00$ per 10 .

$3-4$ ft., $\$ 10.00$ per 10.

S. sanguinea. Reddish purple flowers, very beautiful. $2-3$ ft., $\$ 7.00$ per 10 .

3-4 ft., $\$ 9.00$ per 10 .

\section{TAMARIX. Tamarisk}

T. africana. Tamarix. Beautiful, small-leaved shrub, suggesting a feathery conifer, flesh-colored flowers in May.

2-3 ft., $\$ 5.00$ per 10 .

3-4 ft., $\$ 6.00$ per 10 .

\section{VACCINIUIM}

V. corymbosum. Swamp Blueberry.

18-24 in., $\$ 4.00$ per 10 .

v. pennsylvanicum. Dwarf Blueberry.

Write for prices. 


\section{VIBURNUM. Arrow-Wood, Snowball}

V. acerifolium. Maple-Leaved Arrow-Wood, or Dockmackie. Shrub of low growth, white flowers in flat heads in May, black fruit in Autumn. 2-3 ft., $\$ 7.00$ per 10 .

v. cassinoides. White rod. Flat heads of white flowers in May, followed by black fruit, thick, handsome ovate leaves.

$$
\begin{array}{lll}
2-3 & \text { ft., } \$ 7.00 \text { per } 10 . \\
3-4 & \text { ft., } \$ 8.00 & \text { per } 10 .
\end{array}
$$

v. dentatum. Arrow-Wood. This was used by the Indians for arrow-making. Greenish white flowers, bright blue fruit, sharply toothed bright green leaves, with bronzy-red Autumn coloring.

$2-3$ ft., $\$ 6.00$ per 10.

3.4 ft., $\$ 7.00$ per 10 .

v. lantana. Wayfaring Tree. Large, strong growing shrub, with thick, roundish leaves, large clusters of white flowers in May, followed by red fruit, turning black.

$$
\begin{aligned}
& 2-3 \text { ft., } \$ 7.00 \text { per } 10 \text {. } \\
& \text { 3.4 ft., } \$ 8.00 \text { per } 10 \text {. }
\end{aligned}
$$

V. lentago. Sheep Berry. Sweet Viburnum. Shrub or small tree; fragrant, creamy white flowers, small, sweet, edible fruit, light green handsome leaves.

$$
\begin{array}{llll}
2-3 & \text { ft., } \$ 7.00 & \text { per } 10 . \\
3-4 & \text { ft., } \$ 8.00 & \text { per } 10 .
\end{array}
$$

v. molle. Downy Arrow-Wood. Coarsely toothed leaves; greenish white flowers, blue, oily fruit.

$$
\begin{aligned}
& 2-3 \text { ft., } \$ 6.00 \text { per } 10 \text {. } \\
& \text { 3-4 ft., } \$ 8.00 \text { per } 10 \text {. }
\end{aligned}
$$

v. Opulus. Cranberry 'Tree. Highbush Cranberry. Similar to Snowball in bush and foliage; greenish white Hydrangea-like flowers. Edible red berries, hanging until destroyed by frost.

$$
\begin{aligned}
& \text { 2-3 ft., } \$ 7.00 \text { per } 10 \text {. } \\
& \text { 3-4 ft., } \$ 9.00 \text { per } 10 \text {. }
\end{aligned}
$$

V. Opulus sterilis. Guelder Rose. Snowball. Large, strong growing shrub, with huge globular heads of bloom the latter part of May.

2 -3 ft., $\$ 8.50$ per 10 .

3-4 ft., $\$ 9.00$ per 10 .

v. plicatum. Japanese Snowball. Handsome, dark green plaited foliage, large white flower heads, very showy, superior to the European sort.

$$
\begin{aligned}
& \text { 2-3 ft., } \$ 9.00 \text { per } 10 \text {. } \\
& \text { 3-4 ft., } \$ 10.00 \text { per } 10 \text {. }
\end{aligned}
$$

v. prunifolium. Black Haw, or Plum-Leaved Viburnum. A vigorous growing plant, with strong branches and dark, shining foliage, snow-white flowers in May and June.

$$
\begin{aligned}
& \text { 2-3 ft., } \$ 7.00 \text { per } 10 \text {. } \\
& \text { 3-4 ft., } \$ 9.00 \text { per } 10 \text {. }
\end{aligned}
$$

v. Sieboldii. Large, dark green leaves, white flowers in May.

$$
\begin{array}{llll}
2-3 & \text { ft., } & \$ 7.00 & \text { per } 10 . \\
3-4 & \text { ft., } & \$ 9.00 & \text { per } 10 .
\end{array}
$$

v. tomentosum. Single Japan Snowball. A single form of the Japan Snowball, with dense heads of white flowers, more delicate than the common Guelder Rose.

2-3 ft., \$7.00 per 10 .

$3-4$ ft., $\$ 9.00$ per 10 . 


\section{Hardy Climbing and Trailing Vines}

\section{ACTINIDIA. Silver Thorn}

A. arguta. A Japanese vine with handsome foliage and white flowers, with purple center, very freely produced; edible fruit.

$2-3$ ft., $\$ 6.00$ per 10 .

\section{AMPELOPSIS. Creeping Ivy}

A. Engelmannii. Short jointed form of Virginia Creeper; rapid grower.

2 Yr., $\$ 25.00$ per 100 .

A. quinquefolia. Virginia Creeper. Rapid growing climber, attaching itself to its support, fivefingered leaves, turning brilliant red in Autumn. 2 Yr., $\$ 4.00$ per $10 ; \$ 25.00$ per 100 .

A. Veitchii. Boston Ivy. Small, Ivy-like leaves, overlapping one another so as to form a dense mass; rapid growing, clinging with tiny tendrils. Unequaled for covering brick or frame walls.

3 Yr., XX, $\$ 6.00$ per $10 ; \$ 40.00$ per 100 .

\section{AKEBIA}

A. quinata. Extremely hardy vine, with small dark green leaves hanging on very late; plum colored, fragrant flowers, very free growing.

2 Yr., $\$ 7.50$ per $10 ; \$ 50.00$ per 100 .

\section{ARISTOLOCHIA. Dutchman's Pipe}

A. Sipho. Rapid growing native vine, with handsome, large, light green leaves and oddly-shaped small, yellowish brown flowers, forms a dense shade.

2 Yr., $\$ 15.00$ per 10.

\section{CELASTRUS. Bitter Sweet}

C. scandens. Bitter Sweet Climbing Waxwork. Native twiner, with oval, light green leaves, small yellow flowers and red fruit in orange capsules, hanging long after the leaves; very showy in Fall.

2 Yr., $\$ 4.00$ per $10 ; \$ 30.00$ per 100 .

\section{CLEMATIS}

C. Flammula. Fragrant Clematis. Sweet-scented white flowers in mid-Summer.

2 Yr., $\$ 7.50$ per $10 ; \$ 70.00$ per 100 .

C. paniculata. Japanese Virgin's Bower. A very desirable climber blooming in late Summer, when it produces a mass of small white, fragrant flowers. Hardy and a rapid grower.

2 Yr., $\$ 5.00$ per $10 ; \$ 50.00$ per 100 .

C. virginiana. American Virgin's Bower. Very rapid growing vine, producing a small white flower in August, followed by feathery seeds.

2 Yr., $\$ 25.00$ per 100 . 


\section{DOLICHOS. Kudzu Vine}

D. japonicus.. Quick growing vine with large, beanlike leaves and pinkish violet, pea-shaped flowers.

$$
\$ 8.00 \text { per } 10 \text {. }
$$

\section{EUONYMUS}

E. radicans. An evergreen vine with neat little oval leaves, clings to walls like Ivy. Desirable for rockeries.

2 Yr., $\$ 3.50$ per $10 ; \$ 25.00$ per 100 .

E. radicans variegata. A variety of the above variegated with cream and yellow flowers.

2 Yr., $\$ 4.00$ per $10 ; \$ 30.00$ per 100 .

E. vegetus. A beautiful hardy climbing vine, covered with green foliage through the Winter and a profusion of red berries. One of the best Euonymus.

2 Yr., $\$ 4.00$ per $10 ; \$ 35.00$ per 100 .

\section{HEDERA. Ivy}

H. helix. True or English Ivy. Clinging evergreen, with large, shining dark green leaves. A north exposure is best for it, as it is likely to suffer from sun scald in the Winter if in the full sun. $\$ 5.00$ per $10 ; \$ 40.00$ per 100 .

\section{JASMINUM. Jasmine}

J. nudiflorum. Yellow Jasmine. Yellow flowers in the early Spring, before the leaves appear.

2 Yr., \$5.00 per 10.

\section{LONICERA. Honeysuckle}

L. brachypoda. Japanese Honeysuckle. Very vigorous, nearly evergreen, pale yellow flowers, fading to white; fragrant.

2 Yr., $\$ 4.00$ per $10 ; \$ 25.00$ per 100 .

L. Halliana. Hall's Japan Honeysuckle. One of the best. Pure white, fragrant flowers, changing to yellow, from July to December; foliage hangs on until January.

2 Yr., $\$ 4.00$ per $10 ; \$ 25.00$ per 100 .

L. Heckrotti. Rose-colored flowers with yellow centers, persistent Summer bloomer.

2 Yr., $\$ 6.00$ per 10 .

L. reticulata aurea. Golden-Nettled Chinese Honeysuckle. Attractive variegated form, the leaves veined with gold, bears shearing well, and can be trained on wires to form a charming garden edging.

$\$ 6.00$ per $10 ; \$ 50.00$ per 100 .

\section{LYCIUM. Matrimony Vine}

L..sinensis. Chinese Matrimony Vine. Vine with small, neat leaves and little pink flowers, followed by a highly ornamental red fruit.

2 Yr., $\$ 5.00$ per 10. 


\section{TECOMA. Trumpet Vine}

T. grandiflora. Chinese Trumpet Vine. Very large flowers, salmon yellow, and broad, bell-shaped

corollas.

$\$ 8.00$ per 10 .

T. radicans. Common Trumpet Vine. Large, funnel-shaped flowers orange and scarlet.

$\$ 8.00$ per 10 .

\section{VINCA. Periwinkle or Myrtle}

v. minor. Myrtle, Periwinkle, Trailer, with shining evergreen leaves and porcelain-blue flowers, very hardy.

2 Yr., $\$ 5.00$ per $100 ; \$ 30.00$ per 1000 .

\section{WISTARIA}

W. sinensis. Chinese Wistaria. The well-known sort with graceful drooping clusters of lilac flowers; very hardy and strong growing. $\$ 8.00$ per 10 .

W. sinensis alba. A form with pure white flowers, extremely elegant in appearance. $\$ 8.00$ per 10 .

\section{VITIS. Grape Vines}

v. cordifolia. Frost Grape. Heart-shaped leaves, small black fruit, very sour until frosted. $\$ 10.00$ per 10 .

v. Labrusca. Fox Grape. Large leaves with woolly under surface; large, dark purple fruit. $\$ 10.00$ per 10.

\section{Ground Covering Plants and Trailers}

COPTIS trifolia. Gold Thread shade. $\$ 30.00$ per 1000 .

EPIGAEA repens. Arbutus. $\$ 20.00$ per 100 .

GOULTHERIA procumbens. Wintergreen. $\$ 6.00$ per $100 ; \$ 30.00$ per 1000 .

LYSIMACHIA Nummularia. Yellow Myrtle. $\$ 6.00$ per $100, \$ 30.00$ per 1000 .

MITCHELLA repens. Squawberry. $\$ 6.00$ per $100, \$ 30.00$ per 1000 .

NEPETA Glechoma. Ground Ivy. $\$ 6.00$ per $100, \$ 30.00$ per 1000 .

PACHYSANDRA termalis. 2 Yr., $\$ 4.00$ per $10, \$ 25.00$ per 100 .

RUBUS hispidus. Running Blackberry. $\$ 6.00$ per $100, \$ 30.00$ per 1000 .

THYMUS Serpyllum. Creeping Thyme. $\$ 6.00$ per $100, \$ 30.00$ per 1000 .

VERONICA officinalis. Speedwell. $\$ 6.00$ per $100, \$ 30.00$ per 1000 . 


\section{Hardy Herbaceous and Perennial Plants}

$\$ 3.00$ per $10, \$ 20.00$ per 100 , except where otherwise noted.

ACHILLEA.. In variety. Yellow and red.

ACONITUM.. In variety. Deep blue. $\$ 4.00$ per 10 , $\$ 30.00$ per 100 .

ALTHAEA rosea. In variety. Double and Single, assorted colors.

ALYSSUM. Fragrant, golden yellow.

AMSONIA. In variety. Blue, red, pink and white. ANEMONE. In variety. Red, pink, rose and white. ANTHEMIS. In variety. Yellow.

AQUILEGIA. In variety. White, blue and yellow.

ARABIS alpina. White.

ARMERIA. In variety. White and pink.

ARTEMISIA. In variety. White.

ASCLEPIAS incarnata. Pink.

ASTER. In variety. White, blue, pink, purple and red.

AUBRETIA. In variety. Purple and blue.

BAPTISIA australis. Deep blue.

BELLIS. In variety. Pink and white.

BOCCONIA cordata. Plume Poppy. Spikes of creamy flowers.

BOLTONIA. In variety. Lavender, white and rose. CAMPANULA. In variety. Blue, white and purple. CENTAUREA. In variety. White and rose.

CERASTIUM. In variety. White.

CHRYSANTHEMUMS. Hardy Pompons. In variety and assorted colors.

\section{CLEMATIS. Virgin's Bower}

C. Davidiana. Large cluster of fragrant blue flowers. $\$ 4.00$ per 10 .

C. recta. Pure white flowers. $\$ 5.00$ per $10, \$ 35.00$ per 100.

COREOPSIS. In variety. Yellow.

DELPHINIUM. In variety. Dark and light blue.

DELPHINIUM Belladonna. Dark blue.

DELPHINIUM Hybrids, \$4.00 per 12, \$35.00 per 100.

DIANTHUS. In variety. Red, pink and white.

DICENTRA. In variety. Pink. $\$ 5.00$ per $10, \$ 50.00$ per 100.

DICTAMNUS. In variety. Pink and white, $\$ 5.00$ per $10, \$ 35.00$ per 100 .

DIGITALIS. In variety. White, purple and rose.

ERYNGIUM. In variety. White and blue.

FUNKIA. In variety, and assorted colors, $\$ 4.00$ per $10, \$ 25.00$ per 100 .

GAILLARDIA grandifiora. Yellow.

GERANIUM. In variety. Blue, white and erimson.

GEUM. In variety and assorted colors. 
HERBACEOUS PERENNIAL PLANTS-Continued

GYPSOPHILA. In variety. White.

HELENIUM. In variety. Yellow and old gold.

HELENIUM autumnale. Yellow.

HELIANTHUS. In variety. Yellow.

HELIOPSIS. In variety. Yellow.

HEMEROCALLIS. In variety. Yellow.

HEUCHERA. In variety. Reddish brown and coral red. $\$ 4.00$ per $10, \$ 30.00$ per 100 .

HIBISCUS. In variety. Crimson, white and rose. $\$ 4.00$ per $10, \$ 30.00$ per 100 .

HOLLYHOCKS. (See Althaea rosea).

IBERIS. In variety. White.

IRIS alpina. Mixed colors.

IRIS germanica. In variety. Blue, white, yellow, violet and lavender.

IRIS Kaempferi. Japanese Iris. Named varieties and assorted colors. $\$ 4.00$ per $10, \$ 30.00$ per 100 .

IRIS Kaempferi. Japanese Iris. Mixed colors. $\$ 4.00$ per $10, \$ 25.00$ per 100 .

LATHYRUS. In variety. Pink, white and crimson.

LIATRIS. In variety and assorted colors.

LILIUM BULBS. In variety and colors. Write for price.

LOBELIA cardinalis. Cardinal flowers.

LUPINUS. In variety. Blue, white and rose.

LYCHNIS. In variety. Scarlet, white and rose.

LYSIMACHIA. In variety. Yellow and white.

LYTHRUM. Rose and pink.

MENTHA piperita. Peppermint. Pale purplish flowers.

MONARDA. In variety. Ccarlet and white.

MYOSOTIS. In variety. Blue and white.

OENOTHERA. In variety. White and yellow.

\section{PAEONIA. Peony}

P. Ambrose Verschaffeitii. Double rose. $\$ 8.00$ per 10 .

P. American Beauty. Rose, fragrant. $\$ 7.00$ per 10 .

P. Cc.nte de Diesbache. Early, dark red. $\$ 6.50$ per 10 .

P. Duchess de Nemours. White tinged with yellow. $\$ 8.00$ per 10 .

P. elegans. Pale rose. $\$ 7.00$ per 10 .

P. festiva maxima. Early, pure white, crimson marking.

$\$ 8.00$ per 10 .

P. Festiva alba. White. $\$ 7.00$ per 10 .

P. insignia. Carmine rose. $\$ 7.00$ per 10 .

P. Josephine Parmentier. Pale pink. $\$ 7.00$ per 10 .

P. Lady Bramwell. Medium, silvery pink. $\$ 8.00$ per 10 . 


\section{PAEONIA-Continued}

P. La sublime. Crimson, fragrant. $\$ 7.00$ per 10 .

P. Mme. Calot. Pale Hydrangea-pink, darker shadings in center. $\$ 7.00$ per 10 .

P. Mme. de Verneville. Fine white. $\$ 8.00$ per 10 .

P. Mme. Eliza Bilmon. White, purplish center. $\$ 7.00$ per 10 .

P. Beau Brummell. Clear pink, medium size, compact double. $\$ 7.00$ per 10.

P. Chas. Gosselyn. Blush guard, sulphur center. $\$ 7.00$ per 10 .

P. Duc de Cazes. Bright pink, shaded violet. $\$ 8.00$ per 10.

P. Ne Plus Ultra. Shell-pink. $\$ 8.00$ per 10.

P. New Giant. Large, clear pink. $\$ 8.00$ per 10 .

P. nigricans. Rich shade of red. $\$ 7.00$ per 10 .

P. Perfection. Soft pink, with light center. $\$ 7.00$ per 10 .

P. Queen Victoria. White. $\$ 8.00$ per 10 .

\section{PAEONIA OFFICINALIS (Early Flowering)}

P. officinalis alba. White. $\$ 9.00$ per 10 .

P. officinalis rosea. Rose. $\$ 9.00$ per 10 .

P. officinalis rubra. Crimson. $\$ 9.00$ per 10 .

\section{PAEONIA}

\section{(Single Flowering Japanese)}

P. Avalanche. Deep pink, full center of yellow stamens. $\$ 8.00$ per 10.

P. Geisha. Deep red. $\$ 8.00$ per 10.

P. Savoy. Pure white, outer petals pink. $\$ 8.00$ per 10 .

P. Tokio. Medium size, Chrysanthemum center, petals shell-pink. $\$ 8.00$ per 10.

P. Wang. Chrysanthemum center of eream white petals. $\$ 8.00$ per 10.

PACHYSANDRA terminalis. $\$ 4.00$ per $10, \$ 25.00$ per 100.

PAPAVER. In variety. White, rose and orange.

PENTSTEMON. In variety. Scarlet, white, purple and carmine.

HARDY PHLOX. In variety. Pink, red, rose, orange, white, scarlet, lavender, flame color. 
HERBACEOUS PERENNIAL PLANTS-Continued

PHLOX. Elizabeth Campbell. Salmon-pink. $\$ 4.00$ per $10, \$ 30.00$ per 100 .

DWARF, or CREEPING PHLOX. In varieties and assorted colors.

PHLOX divaricata. In variety.

PHLOX suffruticosa. Miss Lingard. Fine white.

PHYSOSTEGIA. In variety. Pink, white and rose.

PLATYCODON. In variety. Blue.

PLUMBAGO Larpentae. Dwarf blue flowers.

PRIMULA. In variety. Yellow and crimson.

PYRETHRUM. In variety. White.

RUDBECKIA. In variety. Orange and yellow.

SALVIA. In variety. Blue, yellow and crimson.

SCABIOSA. In variety. Lavender, blue and white.

SEDUM. In variety. Yellow, white, rose and pink.

SHASTA DAISY. (See CHRYSANTHEMUM).

SOLIDAGO. In variety. Yellow.

SPIREA. In variety. Pink, white and red.

STOKESIA. In variety. Lavender, and pure white. THALICTRUM. In variety. Yellow and white.

THYMUS. In variety. Red and white.

TRILLIUM grandiflora. Wood Lily. White.

TRITOMA. In variety. Scarlet and red.

TROLLIUS. In variety. Yellow.

VALERIANA. In variety. White and red.

VERONICA. In variety. Blue. $\$ 5.00$ per $10, \$ 35.00$ per 100 .

VINCA minor. $\$ 5.00$ per $100, \$ 30.00$ per 1000 .

VIOLA. In variety. Lavender, white and yellow.

YUCCA filamentosa. Waxy-white, bell-shaped flowers. $\$ 4.00$ per $10, \$ 30.00$ per 100 .

\section{Hardy Ferns}

\section{ADIANTUM. Maiden-Hair Fern}

A. pedatum. Finely divided fronds with ebony-black stems.

$\$ 3.00$ per $10, \$ 8.00$ per 100 .

\section{ASPIDIUM. Christmas Fern}

A. acrostichoides. Christmas Fern. Long, glossy, deep green fronds; evergreen. $\$ 3.00$ per $10, \$ 8.00$ per 100 .

A. marginale. Narrow fronds, $1 / 2 \mathrm{ft}$. long, toothed; damp places. $\$ 3.00$ per $10, \$ 8.00$ per 100 .

A. spinulosum. Thin, finely cut fronds, evergreen. $\$ 3.00$ per $10, \$ 8.00$ per 100 .

A. Thelyteris. $\$ 3.00$ per $10, \$ 8.00$ per 100 .

\section{DICKSONIA. Gossamer Fern}

D. punctilobula. Slenter and finely cut, pale green. $\$ 3.00$ per $10, \$ 8.00$ per 100 . 


\section{OSMUNDA. Cinnamon Fern}

o. cinnamonea. Divided fronds covered with reddish wood when young, very handsome. $\$ 3.00$ per $10, \$ 10.00$ per 100 .

o. Claytoniana. Large, divided fronds, woolly when young.

$\$ 3.00$ per 10, $\$ 12.00$ per 100 .

\section{ONOCLEA}

o. sensibilis. Sensitive Fern. $\$ 3.00$ per $10, \$ 8.00$ per 100 .

o. Struthiopteris. Ostrich Fern. $\$ 3.00$ per $10, \$ 10.00$ per 100 .

\section{POLYPODIUM}

P. vulgare. Polypody. Evergreen variety, well suited for rockeries.

$\$ 3.00$ per $10, \$ 8.00$ per 100 .

\section{Ornamental Grasses}

\section{EULALIA}

E. gracillima. A very handsome Japanese grass, forming a broad clump, narrow dark green leaves, with silver white midrib. Bears a feathery plume of flowers in Autumn.

$\$ 4.00$ per $10, \$ 30.00$ per 100 .

E. japonica. Tall grass with plain green leaves, attaining a height of 5 to $7 \mathrm{ft}$. $\$ 3.00$ per $10, \$ 25.00$ per 100 .

E. zebrina. Zebra Grass. Leaves barred with bright yellow, very striking.

$\$ 4.00$ per 10, \$3.00 per 100.

\section{PHLARIS. Ribbon Grass}

P. arundinacea variegata. Large variegated foliage, valuable for edging and large beds. $\$ 4.00$ per $10, \$ 30.00$ per 100 .

\section{Roses}

\section{HARDY CLIMBING ROSES}

In units of 10. $\$ 7.50$ per $10, \$ 65.00$ per 100 , except where otherwise noted.

American Pillar. Rosy pink, single. $\$ 8.00$ per 10 , $\$ 65.00$ per 100 .

Christine Wright. Bright pink. $\$ 9.00$ per $10, \$ 75.00$ per 100.

Crimson Rambler. Deep crimson. $\$ 7.00$ per $10, \$ 55.00$ pèr 100.

Dorothy Perkins. Pink. $\$ 5.00$ per $10, \$ 40.00$ per 100.

Dr. Van Fleet. Rich flesh pink. $\$ 7.00$ per $10, \$ 65.00$ per 100.

Excelsa. Clear scarlet. $\$ 5.00$ per $10, \$ 40.00$ per 100 . 


\section{ROSES-Continued}

Gardenia. Bright yellow. $\$ 6.00$ per $10, \$ 50.00$ per 100.

Hiawatha. Crimson with white eye, single. $\$ 6.00$ per 10, $\$ 45.00$ per 100 .

Lady Gay. Pink. $\$ 6.00$ per $10, \$ 45.00$ per 100 .

Pauls Scarlet. Vivid scarlet. $\$ 9.00$ per $10, \$ 75.00$ per 100.

Silver Moon. Silvery white. $\$ 9.00$ per $10, \$ 75.00$ per 100 .

Tausendschon. Varies from white to pink. $\$ 6.00$ per $10, \$ 50.00$ per 100 .

White Dorothy Perkins. White. $\$ 6.00$ per $10, \$ 50.00$ per 100.

Wichuriana. Single white, trailing. $\$ 6.00$ per 10 , $\$ 50.00$ per 100 .

\section{DWARF POLYANTHA or "BABY" ROSES}

In units of 10 . $\$ 9.00$ per $10, \$ 75.00$ per 100 .

Baby Rambler. Pink.

Baby Rambler. Crimson.

Baby Tausendschon. Pink.

Ellen Poulson. Dark pink.

Greta Kluis. Red.

Jessie. Scarlet.

Miss Edith Cavell. Brilliant erimson.

Orleans. Brilliant red.

Phyllis. Dark carmine pink.

Baby Dorothy. Pink.

\section{HYBRID TEA AND EVER- BLOOMING ROSES}

In units of 10 . $\$ 9.00$ per $10, \$ 75.00$ per 100 , except where otherwise noted.

Bessie Brown. Flesh pink.

Chatteau de Cose Bouget. Velvety scarlet.

Cheerful. Orange.

Columbia. Glowing double pink. (New). $\$ 10.00$ per $10, \$ 90.00$ per 100 .

Crusader. Crimson. (New). $\$ 10.00$ per $10, \$ 90.00$ per 100.

Dean Hole. Light carmine.

Duchess of Wellington. Saffron yellow. $\$ 10.00$ per $10, \$ 90.00$ per 100 .

George Ahrends. Pink.

George C. Waud. Glowing orange crimson.

Golden Emblem. Deep golden yellow. $\$ 15.00$ per 10 .

Gorgeous. Deep orange yellow. $\$ 10.00$ per $10, \$ 90.00$ per 100 .

Gruss an Teplitz. Scarlet.

Hadley. Velvety crimson. $\$ 9.00$ per $10, \$ 85.00$ per 100.

J. J. L. Mock. Deep pink.

Juliet. Old gold and red.

Killarney. Pink. 


\section{ROSES-Continued}

Konigan Carola. Satiny rose.

LaFrance. Rose pink.

Lady Ashtown. Pale rose shading to yellow.

Lady Hillingdon. Deep apricot yellow. $\$ 9.00$ per 10 , $\$ 85.00$ per 100 .

Laurent Carle. Deep rosy crimson.

Los Angeles. Flame pink toned with coral. $\$ 15.00$ per $10, \$ 125.00$ per 100 .

Mme. Caroline Testout. Clear rich pink.

Mme. Edouard Herriott. Coral red shaded with yellow. Maman Cochet. Pink.

Miss Cynthia Forde. Brilliant rose pink.

Miss Lolita Armour. Deep coral red. $\$ 15.00$ per 10 , $\$ 125.00$ per 100 .

Mrs. George Shawyer. Brilliant rose pink.

old Gold. Reddish orange. $\$ 10.00$ per $10, \$ 85.00$ per 100.

Ophelia. Salmon pink shaded with rose. $\$ 10.00$ per $10, \$ 85.00$ per 100 .

Premier. Clear shade of rose pink. (New). $\$ 15.00$ per 10, $\$ 125.00$ per 100 .

Radiance. Carmine pink.

Sunburst. Rich yellow shaded to coppery orange. $\$ 10.00$ per $10, \$ 90.00$ per 100 .

White Cochet. Snow white.

White Killarney. Waxy white. $\$ 10.00$ per $10, \$ 90.00$ per 100.

William F. Dreer. Shell pink, yellow at base. $\$ 15.00$ per 10, $\$ 125.00$ per 100 .

Willowmere. Shrimp pink shaded to yellow and car. mine. $\$ 10.00$ per $10, \$ 85.00$ per 100 .

\section{HYBRID PERPETUAL ROSES}

In units of 10 . $\$ 7.50$ per $10, \$ 65.00$ per 100 , except where otherwise noted.

American Beauty. Crimson. $\$ 9.00$ per $10, \$ 75.00$ per 100.

Black Prince. Dark crimson.

Captain Christy. Flesh pink.

Clio. Flesh color.

Eugene Furst. Deep crimson.

Frau Karl Druschki. Very best, large white roses.

General Jacqueminot. Bright crimson.

George Ahrends. Pink.

John Hopper. Rose pink.

Magna Charta. Pink, large.

Margaret Dickson. White with flesh centre.

Marshall P. Wilder. Crimson, extra large.

Paul Neyron. Pink shading to rose, very large.

Ulrich Brunner. Light red, large eup shape.

\section{STANDARD OR TREE ROSES}

In units of 10 . Our assortment. Assorted colors $2-2 \frac{1}{2}$ ft., $\$ 25.00$ per $10, \$ 200.00$ per 150. 


\section{Fruit Trees}

\section{APPLE TREES}

4-6 ft., first class, $\$ 10.00$ per $10, \$ 75.00$ per 100 . 6-7 ft., $X, \$ 15.00$ per 10 , except where otherwise noted.

\section{SUMMER VARIETIES}

Early Harvest. Pale yellow, acid.

Early Strawberry. Red, sub-acid.

Sweet Bough. Yellow, sweet.

Red Astrachan. Red, acid.

Summer Rambo. Red, sub-acid.

Yellow Transparent. Yellow, sub-acid.

\section{AUTUMN VARIETIES}

Cornell's Fancy. Red, sub-acid.

Duchess of Oldenburg. Yellow, streaked red, subacid.

Fall Pippin. Yellow, sub-acid.

Gravenstein. Striped and splashed red on yellow, subacid.

Jersey Sweet. Striped red on greenish yellow, sweet. Maiden's Blush. Yellow, red eheek, sub-acid.

\section{WINTER VARIETIES}

Baldwin. Red, sub-acid.

Ben Davis. Red, acid.

Delicious. Red, sub-acid.

Grimes Golden. Yellow, sub-acid.

Jonathan. Red, sub-acid.

Northern Spy. Striped red, sub-acid.

Paragon. Red, sub-acid.

R. I. Greening. Green, ripening yellow, acid.

Roxbury Russet. Greenish russet, sub-acid.

Rome Beauty. Red, sub-acid.

Stayman's Winesap. Red, acid.

Wealthy. Yellowish, shaded dark red, sub-acid.

Winter Banana. Sub-acid.

York Imperial. Whitish, shaded crimson, sub-acid.

\section{CHERRY TREES}

\section{SWEET CHERRIES}

4-6 ft., first class, $\$ 17.50$ per 10, $\$ 150.00$ per 100 .

Black Tartarian. Very large, purplish black, June to July.

Gov. Wood. Light red. End of June.

Napoleon Bigarreau. Pale yellow, red check. Late.

Windsor. Black, overspread red, valuable. July.

Yellow Spanish. Very large, pale yellow, red eheck; one of the best.

\section{SOUR CHERRIES}

4-5 ft., first class, $\$ 17.50$ per $10, \$ 150.00$ per 100 .

English Morello. Large dark red, becoming nearly black. After mid-Summer.

Early Richmond. Clear red, very productive. Early.

Montmorency. Large, bright red, very hardy and productive. Medium. 


\section{PEACH TREES}

4-5 ft., first class, $\$ 7.50$ per $10, \$ 50.00$ per 100 . 5-6 ft., $X, \$ 10.00$ per $10, \$ 90.00$ per 100 .

Belle of Georgia. White, red cheek. August.

Carman. Large, creamy white, splashed red. Medium.

Champion. Creamy white, red cheek, white flesh. Early to midseason.

Crawford's Late. Very fine and yellow. End of September.

Elberta. Lemon yellow, red blush, yellow flesh. Medium late.

Fox seedling. White freestone, good for shipping. Medium.

Greensboro. Red, shaded yellow, white flesh. Early.

J. H. Hale. Red, white flesh. Mid-season.

old Mixon Free. Greenish white and red, hardy. Early.

Smock. Large, bright yellow and orange. Late.

- Stump the World. Red and white, handsome, productive. End of September.

\section{PEAR TREES}

4-6 ft., first class, $\$ 15.00$ per $10, \$ 125.00$ per 100 .

\section{SUMMER VARIETIES}

Bartlett. Large yellow; one of the best; very juicy and highly flavored. August and September.

Clapp's Favorite. Large, yellow, dotted red. Ripens just before Bartlett.

Wilder. Small, greenish yellow with reddish cheeks; very good; sub-acid. First of August.

\section{AUTUMN VARIETIES}

Beurre d'Anjou. Large, russety yellow, delicious. September.

Duchess d'Angouleme. Very large, greenish yellow, juicy and excellent. October and November.

Howell. Large, yellow, delicious, sweet and melting. September and October.

Kieffer. Large golden yellow, dotted brown, good for canning. October and November.

Manning's Elizabeth. Medium size, bright yellow, red cheek, flesh white, juicy, melting. September.

Seckel. Small but very juicy and rich. September.

Sheldon. Round, russet, rich and melting. October.

\section{WINTER VARIETIES}

Lawrence. Large, golden yellow, high quality, buttery, rich and aromatic. Keeps until March.

Mount Vernon. Medium to large, skin yellow, russet color, juicy, aromatic. November to January.

Winter Nellis. One of the best early Winter pears, rich and buttery and a good bearer. Keeps until March.

\section{DWARF PEAR TREES}

\section{3-4 ft., $\$ 12.50$ per $10, \$ 100.00$ per 100 .}

\section{VARIETIES}

Bartlett, Clapp's Favorite, Beurre d'Anjou, Kieffer, Seckel, Duchess d'Angouleme and Howell. 


\section{PLUM TREES}

4-5 ft., first class, $\$ 15.00$ per $10, \$ 125.00$ per 100 .

Bradshaw. Dark violet red, very productive. Early. Coe's Golden Drop. Large, bright yellow. Last of September.

German Prune. Medium, purple or blue. September. Imperial Gage. Large, greenish, delicious. Middle of August.

Lombard. Violet red, very hardy and productive. September.

Yellow Gage. Yellow, rich, productive. Middle of August.

\section{JAPANESE VARIETIES}

Abundance. Amber turning to red. Early.

Burbank. Orange yellow, suffused red, mid-season to late.

Climax. Dark purple, large and handsome.

Ogon. Golden yellow, freestone, fine.

Red June. Bright red. Second early.

Shipper's Pride. Purple, excellent. Mid-season.

\section{Small Fruits}

\section{BLACKBERRY BUSHES}

$\$ 12.00$ per 100 .

VARIETIES

Eldorado. Jet black.

Erie. Large and productive, tender.

Kittatinny. Large roundish fruit, juicy, sweet.

Rathbun. A new variety. Berries extra large, jet black, quality good.

\section{CURRANTS}

2 Yr., $\$ 4.00$ per 10. $\$ 25.00$ per 100 .

Cherry. Red.

\section{VARIETIES}

Fay's Prolific. Red.

Perfection. A new variety, mild, sub-acid. $\$ 5.00$ per $10, \$ 35.00$ per 100 .

White Grape. White.

\section{GOOSEBERRIES}

2 Yr., $\$ 4.00$ per $10, \$ 30.00$ per 100 .

Houghton. Red.

Joselyn. Red Jacket. Red.

Downing. Fine American sort, whitish green.

\section{GRAPES}

2 Yr., $\$ 5.00$ per $10, \$ 30.00$ per 100 , except where otherwise noted.

Agawam. Red.

Brighton. Coppery Red.

Campbell's Early. Black.

Catawba. Coppery Red. 
Champion. Black.

\section{GRAPES-Continued}

Concord. Black.

Delaware. Red.

Moore's Diamond. Yellowish green.

Moore's Early. Black.

Niagara. White.

Wilder. Black.

Worden. Black.

\section{RASPEBRRIES}

$\$ 12.00$ per 100, except where otherwise noted.

\section{VARIETIES}

Brandywine. Bright scarlet.

Columbian. Large, purple.

Cumberland. Black.

Cuthbert. Crimson, large.

Golden Queen. Light yellow.

LaFrance. Everbearing. $\$ 7.50$ per $10, \$ 40.00$ per 100 .

Loudon. Red.

Miller Red. Red.

St. Regis. Everbearing. $\$ 15.00$ per 100 .

\section{STRAWBERRIES \\ EVERBEARING VARIETIES \\ $\$ 5.00$ per 100 .}

ORDINARY VARIETIES

$\$ 2.00$ per 100 .

\section{ASPARAGUS}

Strong 2-Yr. roots, $\$ 4.00$ per $100, \$ 25.00$ per 1000 .

VARIETIES

Barr's Mammoth, Conover's Colossal and Palmetto.

\section{RHUBARB}

2 Yr. old, $\$ 30.00$ per 100 .

Forcing clumps, $\$ 50.00$ per 100 .

\section{GRASS SEED}

For all purposes. Send list of wants for price.

For trees of larger size and quantities not given herein, kindly send list of wants, stating quantity and size for estimate. 


\section{INDEX}

$$
-\mathrm{A}-
$$

Name

Page

Abelia ............................ 17

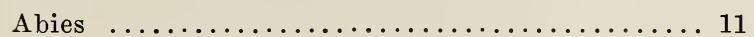

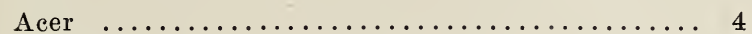

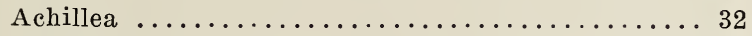

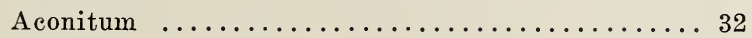

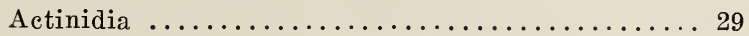

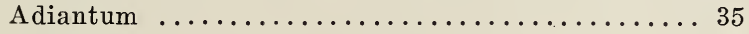

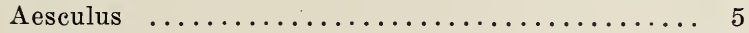

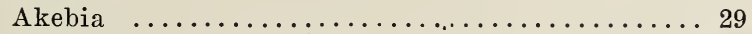

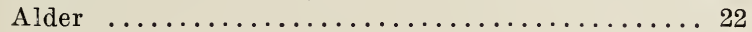

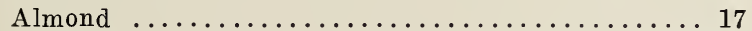

Althaea $\ldots \ldots \ldots \ldots \ldots \ldots \ldots \ldots \ldots \ldots \ldots \ldots 21,32$

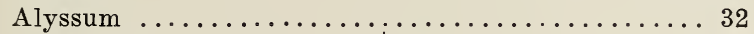

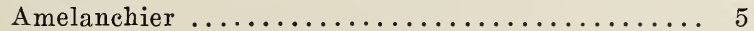

Amorpha ............................ 17

Ampelopsis ..............................29

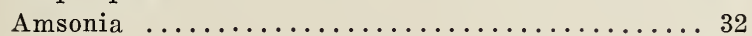

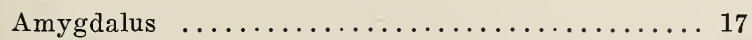

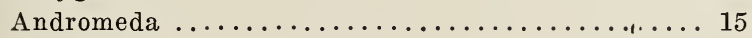

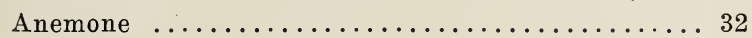

Angelica Tree $\ldots \ldots \ldots \ldots \ldots \ldots \ldots \ldots \ldots \ldots, 17$

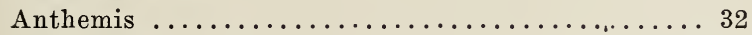

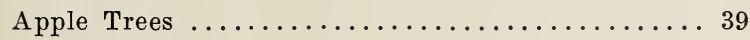

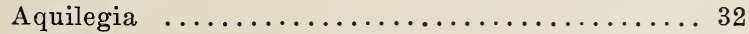

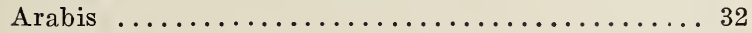

Aralia ...................... 5, 17

Arborvitae ....................11, 14

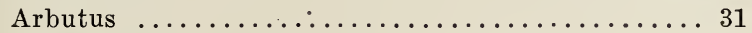

Aristolochia ......................... 29

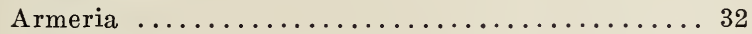

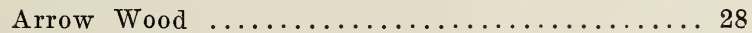

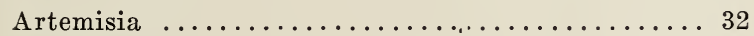

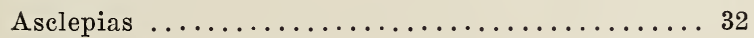

Ash ............................... 7

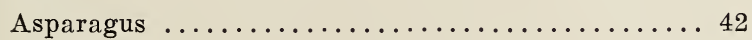

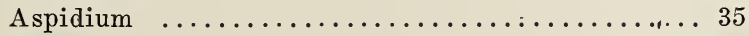

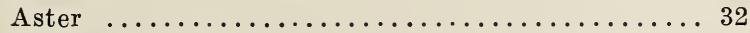

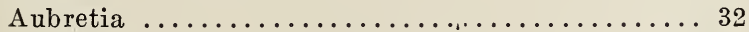

Azalea .................. 15, 16, 17

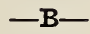

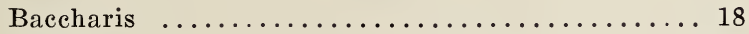

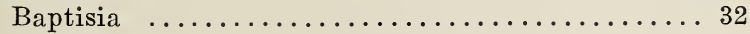

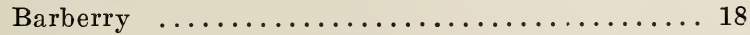

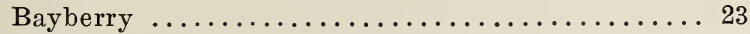

Beech ........................... 7

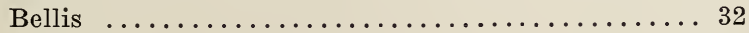

Bensoin .............................. 18

Berberis ............................ 18 
Betula ........................ 5

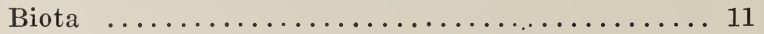

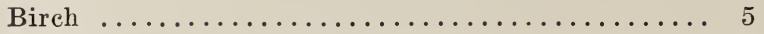

Bitter Sweet ...................... 29

Blackberry Bushes ................... 41

Blackberry, Running ................. 31

Bladder Senna ..................... 19

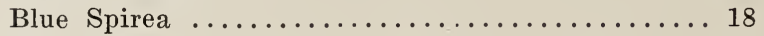

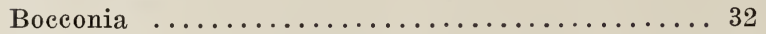

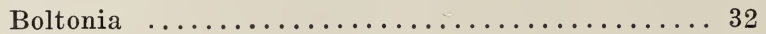

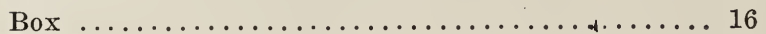

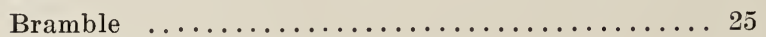

Buckthorn ....................... 24

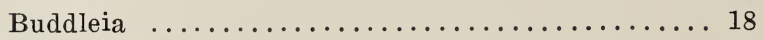

Burning Bush ...................... 21

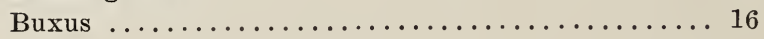

Callicarpa ............................ 18

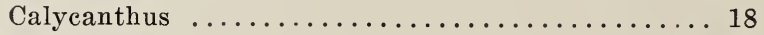

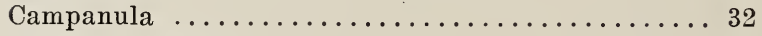

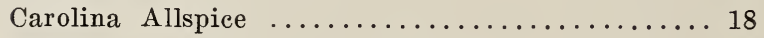

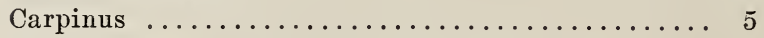

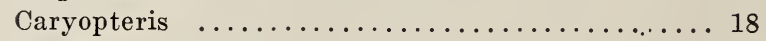

Catalpa .......................... 5

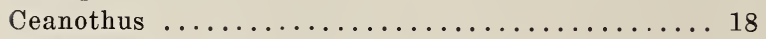

Celastrus ........................ 29

Celtis .......................... 6

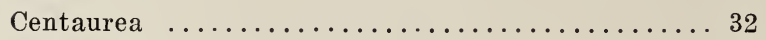

Cerastium ...................... 32

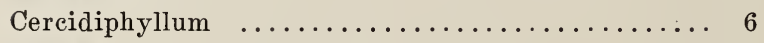

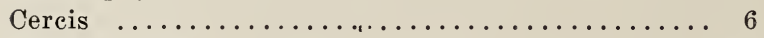

Cherry Trees ...................... 39

Chionanthus ...................... 19

Christmas Fern ................... 35

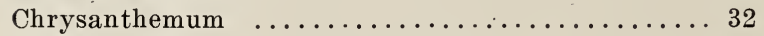

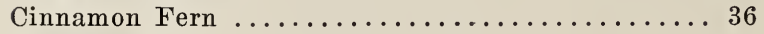

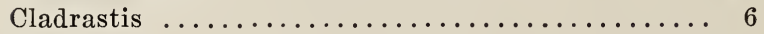

Clematis . .................... 29, 32

C. Davidiana ..................... 32

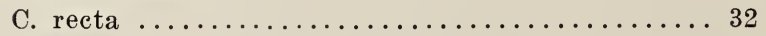

Clethra ........................ 19

Coffee Tree ...................... 7

Colutea ........................... 19

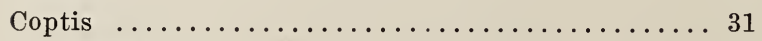

Coral Berry .................... 26

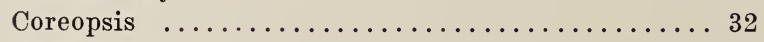

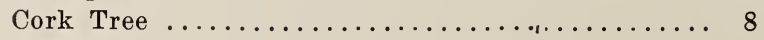

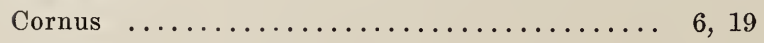

Corylus .......................... 19

Cotoneaster ...................... 16

Crab Apple ........................ 9

Crataegus $\ldots \ldots \ldots \ldots \ldots \ldots \ldots \ldots \ldots \ldots$ 6. 7 
Name

Page

Creeping Ivy ....................... 29

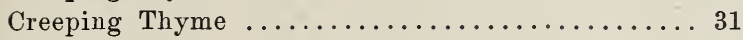

Currants ........................4 41

Cydonia .......................... 19

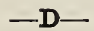

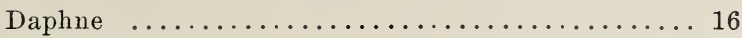

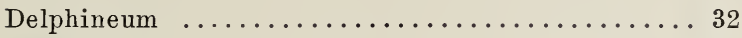

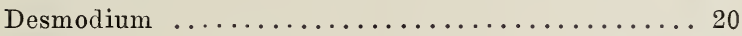

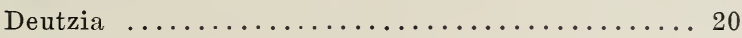

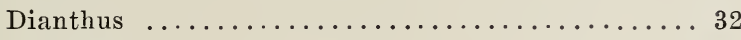

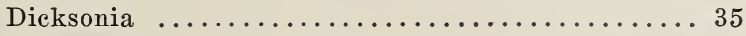

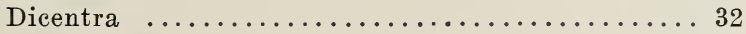

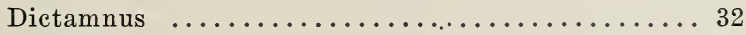

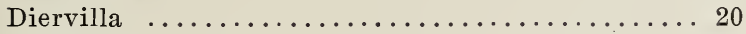

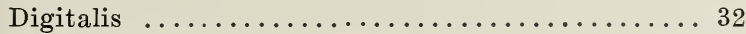

Dogwood ...................... 6, 19

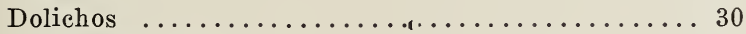

Dutchman's Pipe .................... 29

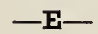

Elaeagnus ....................... 20

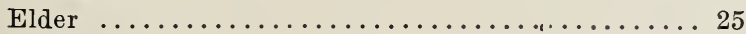

$\operatorname{Elm} \ldots \ldots \ldots \ldots \ldots \ldots \ldots \ldots \ldots \ldots \ldots \ldots \ldots$

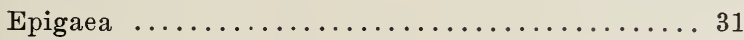

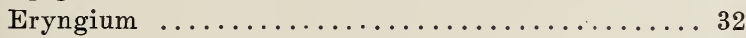

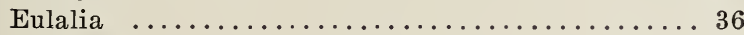

Euonymus .................. 16, 21, 30

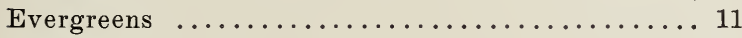

Exochorda $\ldots \ldots \ldots \ldots \ldots \ldots \ldots \ldots \ldots \ldots \ldots \ldots$

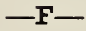

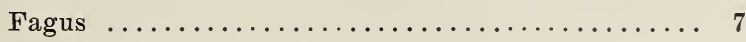

False Indigo $\ldots \ldots \ldots \ldots \ldots \ldots \ldots \ldots \ldots \ldots \ldots \ldots$

Ferns, Hardy ................... 35, 36

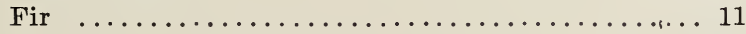

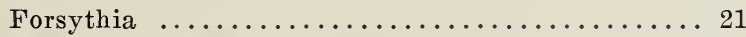

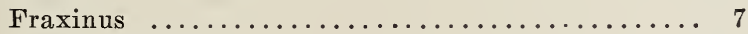

French Mulberry .................. 18

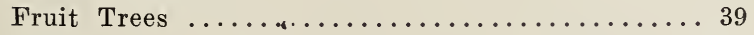

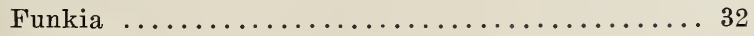

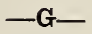

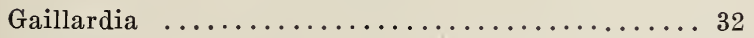

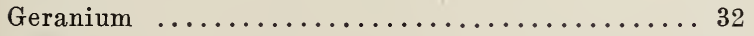

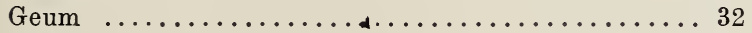

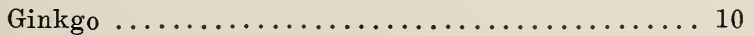

Golden Bell ........................ 21

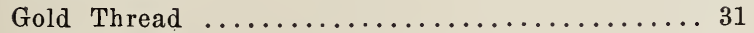

Gooseberries .......................41

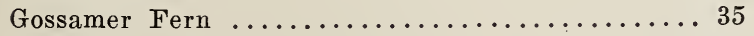

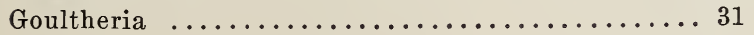




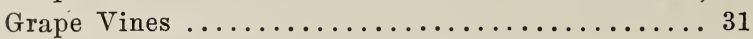

Grasses, Ornamental ................. 36

Grass Seed ...................... 42

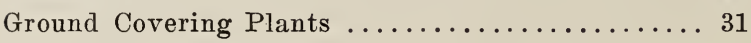

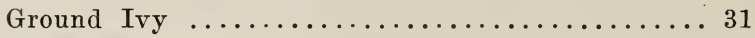

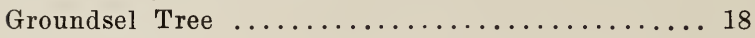

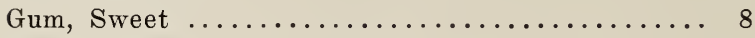

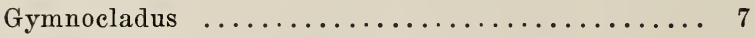

Gypsophilla $\ldots \ldots \ldots \ldots \ldots \ldots \ldots \ldots \ldots \ldots \ldots \ldots$

\section{$-\mathbf{H}-$}

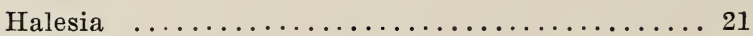

Hamamelis ...................... 21

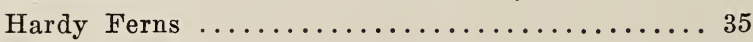

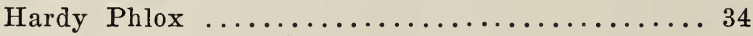

Hazel ......................... 19

Hedera ......................... 30

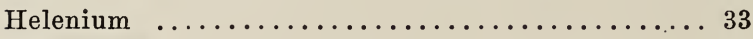

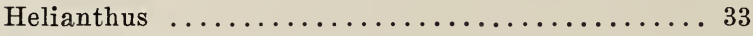

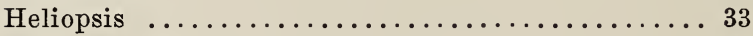

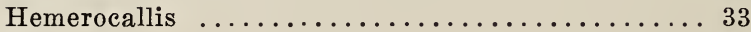

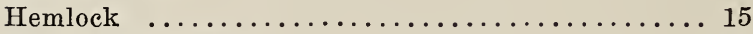

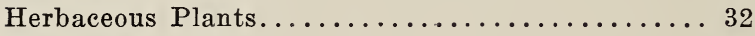

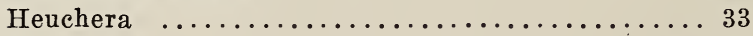

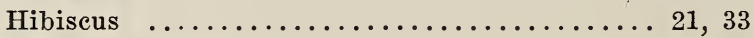

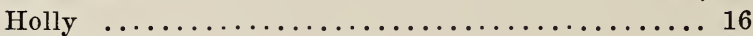

Hollyhocks ....................... 33

Honeysuckle .................... 23, 30

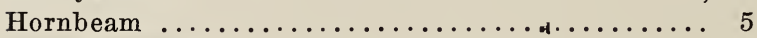

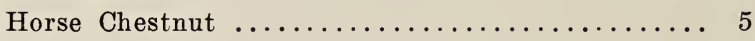

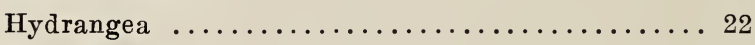

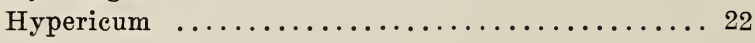

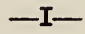

Iberis $\ldots \ldots \ldots \ldots \ldots \ldots \ldots \ldots \ldots \ldots \ldots \ldots \ldots \ldots \ldots$

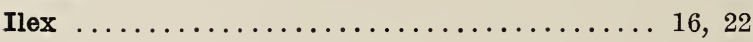

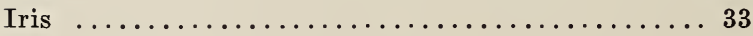

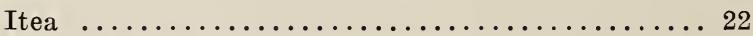

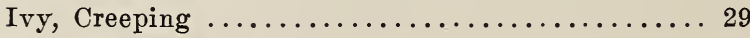

Ivy, Ground ........................... 31

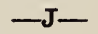

Japan Cypress ..................... 13

Japan Maple ....................... 5

Japanese Quince ..................... 19

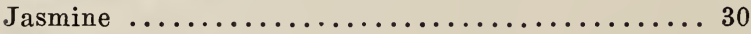

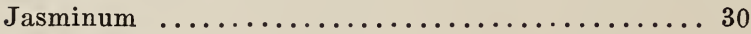

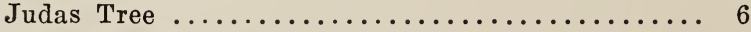

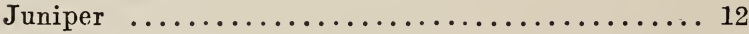

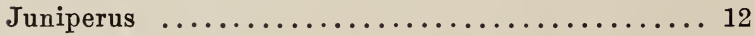


$-\mathbf{K}-$

Name Page

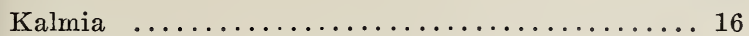

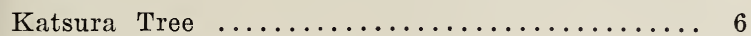

Koelreuteria $\ldots \ldots \ldots \ldots \ldots \ldots \ldots \ldots \ldots \ldots \ldots \ldots$

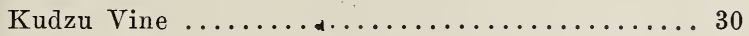

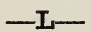

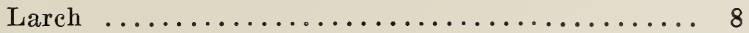

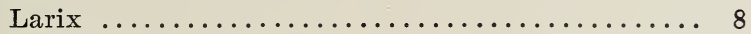

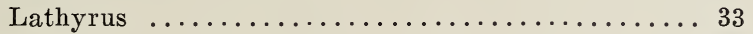

Laurel ........................... 16

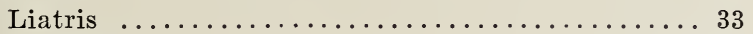

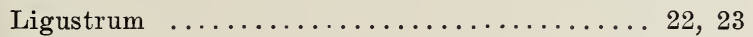

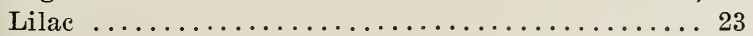

Lilium Bulbs ....................... 33

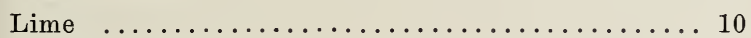

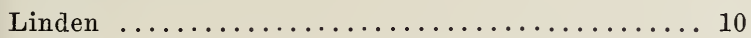

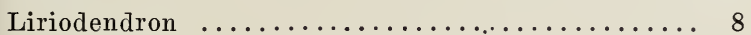

Liquidamber $\ldots \ldots \ldots \ldots \ldots \ldots \ldots \ldots \ldots \ldots \ldots \ldots$

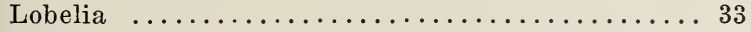

Lonicera ..................... 23, 30

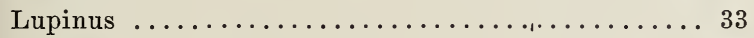

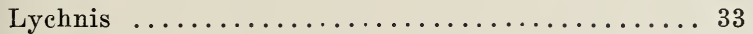

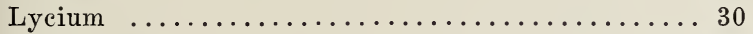

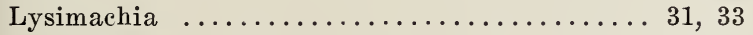

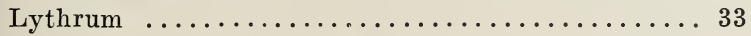

$-\mathbf{M}-$

Magnolia .......................... 8

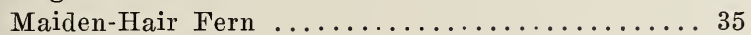

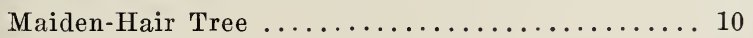

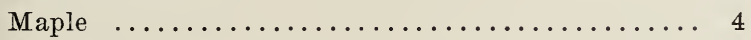

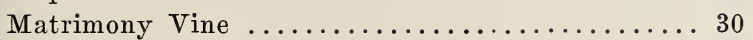

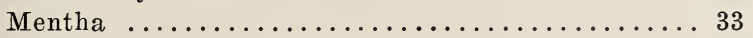

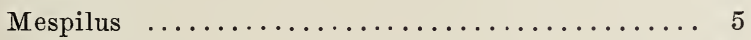

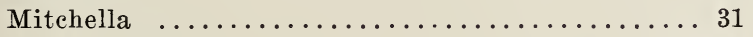

Mock Orange .................. 23, 24

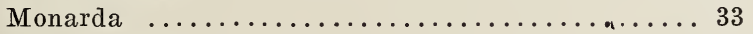

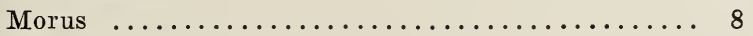

Mountain Holly ......................... 22

Mulberry ....................... 8

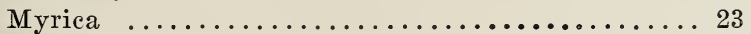

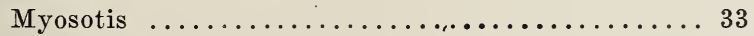

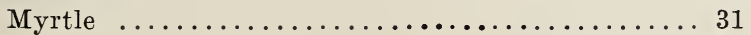

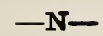

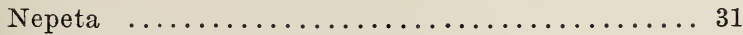

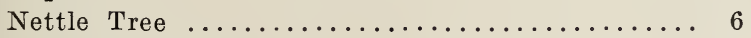

New Jersey Tea ..................... 18

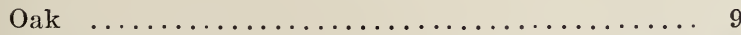

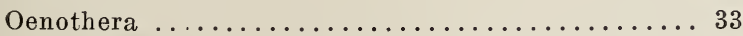


Name

Page

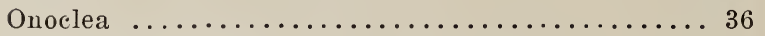

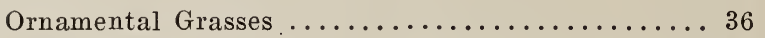

Osmunda ............................. 36

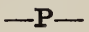

Pachysandra ..................... 31, 34

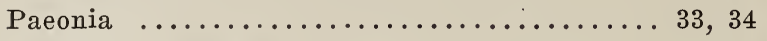

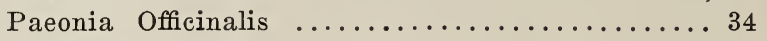

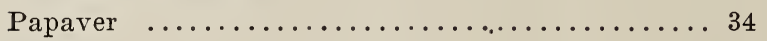

Peach ............................. 8

Peach Trees ......................... 40

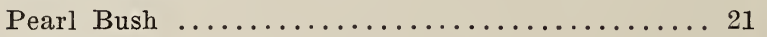

Pear Trees ..............................40 40

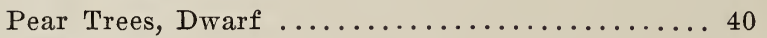

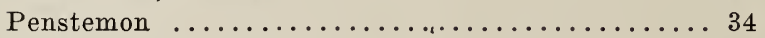

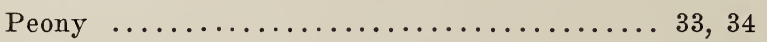

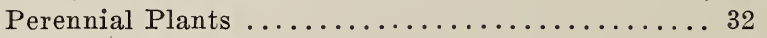

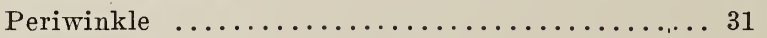

Persica ........................ 8

Phellodendron $\ldots \ldots \ldots \ldots \ldots \ldots \ldots \ldots \ldots \ldots . \ldots \ldots$

Philadelphus .................... 23, 24

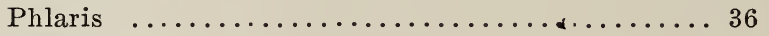

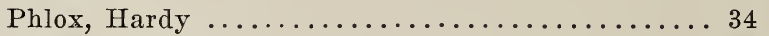

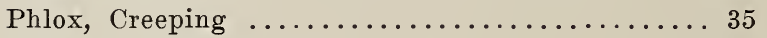

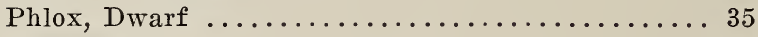

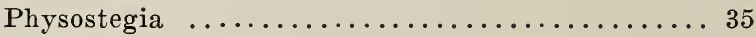

Picea ........................ 12, 13

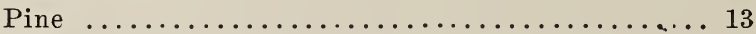

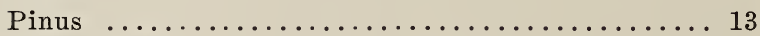

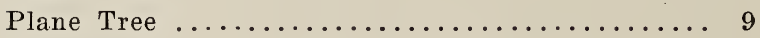

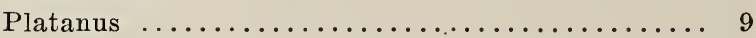

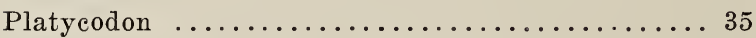

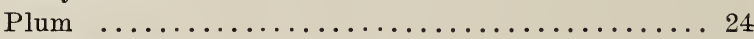

Plum Trees $\ldots \ldots \ldots \ldots \ldots \ldots \ldots \ldots \ldots \ldots \ldots 41$

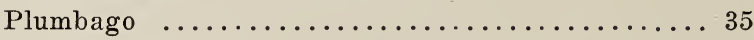

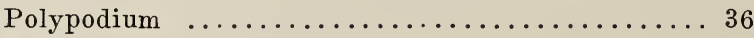

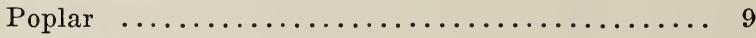

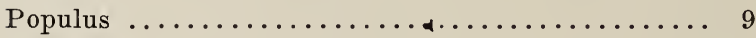

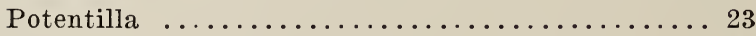

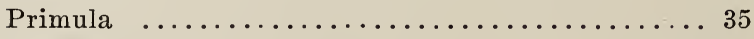

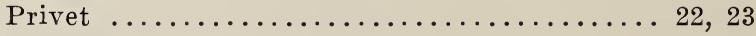

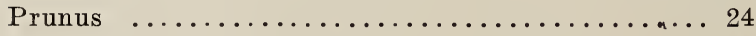

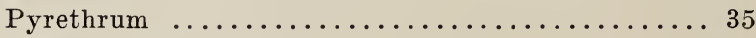

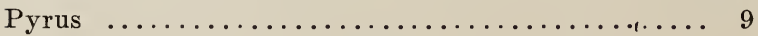

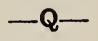

Quercus

Raspberries 
Rhodotypos ...................... 24

Rhubarb ...................... 42

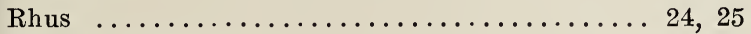

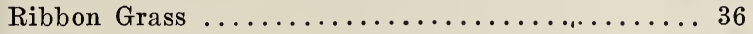

Roses ...................... 36, 38

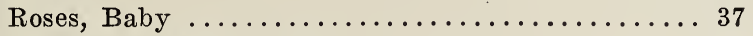

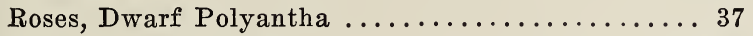

Roses, Everblooming ............. 37, 38

Roses, Hardy Climbing ............. 36, 37

Roses, Hardy Perpetual ................. 38

Roses, Hybrid Tea ............... 37, 38

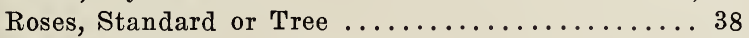

Rose of Sharon ..................... 21

Rubus ...................... 25, 31

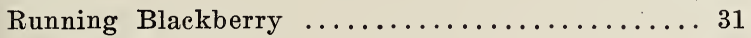

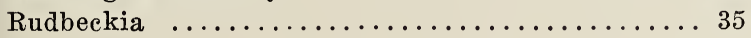

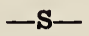

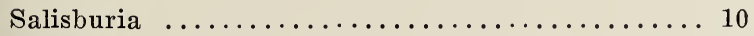

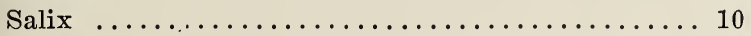

Salvia .......................... 35

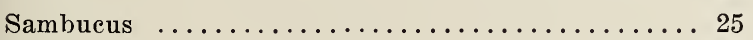

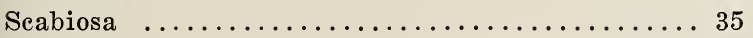

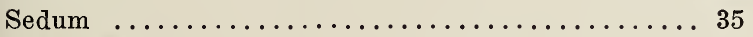

Shade Trees ...................... 4

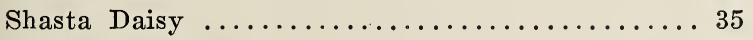

Shrubs, Deciduous .................. 17

Shrubs, Evergreen ................... 15

Shrubby Cinquefoil ................ 23

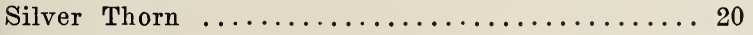

Small Fruits ..................... 41

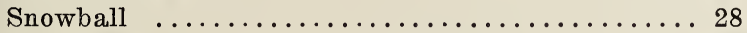

Snowberry ...................... 26

Snowdrop Tree ..................... 21

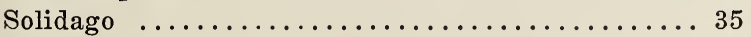

Sorbus ......................... 10

Speedwell ......................... 31

Spindle Tree ...................... 21

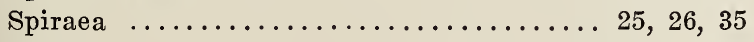

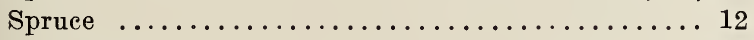

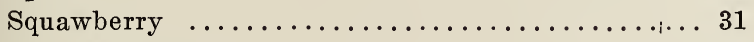

St. John's Wort ................... 22

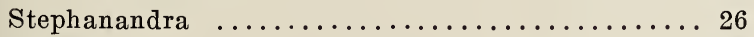

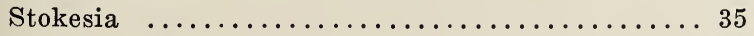

Strawkerries ..................... 42

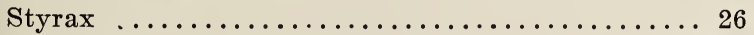

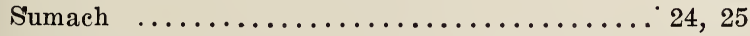

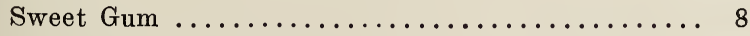

Sweet Pepper Bush .................. 19

Symphoricarpos .................. 26

Syringa $\ldots \ldots \ldots \ldots \ldots \ldots \ldots \ldots \ldots \ldots \ldots \ldots \ldots \ldots \ldots \ldots \ldots$ 
Name

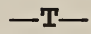

Tamarisk $\ldots \ldots \ldots \ldots \ldots \ldots \ldots \ldots \ldots \ldots \ldots \ldots .27$

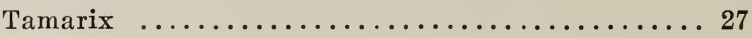

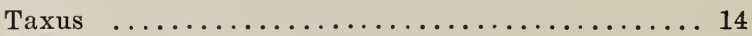

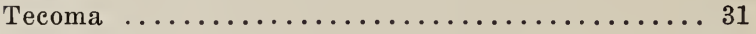

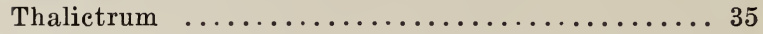

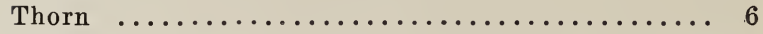

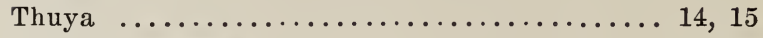

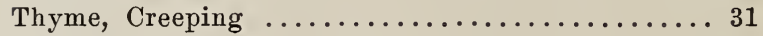

Thymus ............................ 31,

Tilia ........................ 10, 11

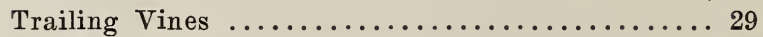

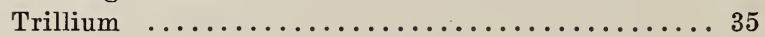

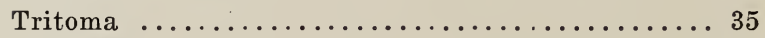

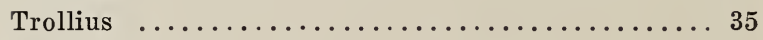

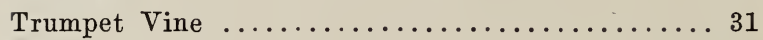

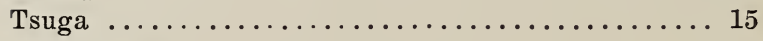

Tulip Tree $\ldots \ldots \ldots \ldots \ldots \ldots \ldots \ldots \ldots \ldots \ldots \ldots .6$

Ulmus

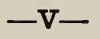

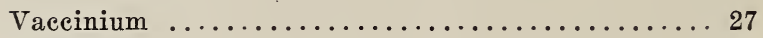

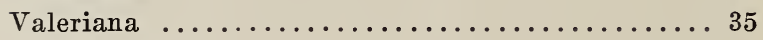

Varnish Tree ........................ 7

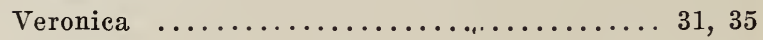

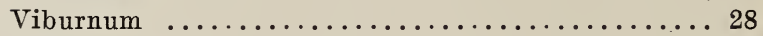

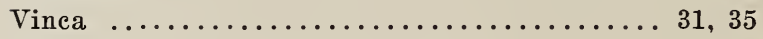

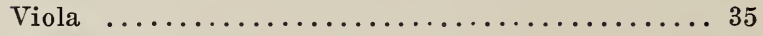

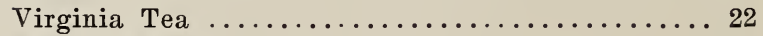

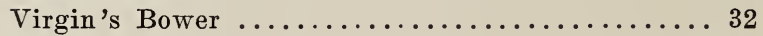

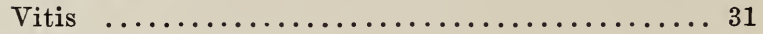

Vines, Hardy Climbing ................. 29

Vines, Trailing .................... 29

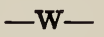

Weigela ............................. 20

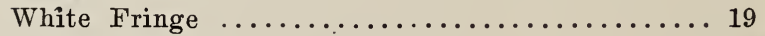

White Kerria ........................ 24

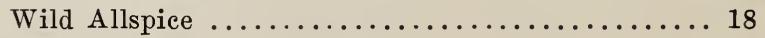

Willow .............................. 10

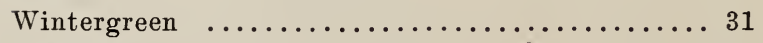

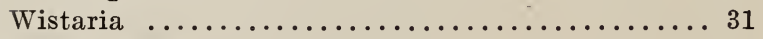

Witeh Hazel ....................... 21

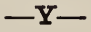

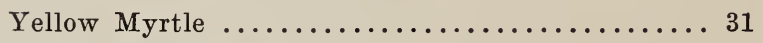

Yellow Wood Tree ..................... 6

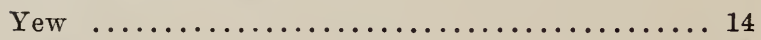

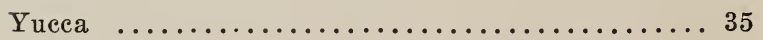




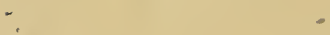

a 